\title{
WestVirginiaUniversity
}

THE RESEARCH REPOSITORY @ WVU

Graduate Theses, Dissertations, and Problem Reports

2014

\section{A hypermedia design for both high and low knowledge readers}

Jonathan Vickers

West Virginia University

Follow this and additional works at: https://researchrepository.wvu.edu/etd

\section{Recommended Citation}

Vickers, Jonathan, "A hypermedia design for both high and low knowledge readers" (2014). Graduate Theses, Dissertations, and Problem Reports. 506.

https://researchrepository.wvu.edu/etd/506

This Thesis is protected by copyright and/or related rights. It has been brought to you by the The Research Repository @ WVU with permission from the rights-holder(s). You are free to use this Thesis in any way that is permitted by the copyright and related rights legislation that applies to your use. For other uses you must obtain permission from the rights-holder(s) directly, unless additional rights are indicated by a Creative Commons license in the record and/ or on the work itself. This Thesis has been accepted for inclusion in WVU Graduate Theses, Dissertations, and Problem Reports collection by an authorized administrator of The Research Repository @ WVU. For more information, please contact researchrepository@mail.wvu.edu. 
A Hypermedia Design For Both High and Low Knowledge Readers Jonathan Vickers

Thesis submitted

to the P.I. Reed School of Journalism

at West Virginia University

in partial fulfillment of the requirements for the degree of

Master of Science in Journalism

\author{
Bob Britten, Ph.D., Chair \\ John Temple, M.F.A. \\ Mary Kay McFarland, M.A. \\ Jeff Jackson, M.A. \\ Department of Journalism
}

Morgantown, West Virginia

2014

Keywords: hypermedia, design, multimedia, prompting, knowledge, readers, websites, nonlinear, storytelling, engagement, interactive

Copyright 2014 Jonathan Vickers 


\begin{abstract}

\section{A Hypermedia Design For Both High and Low Knowledge Readers}

\title{
Jonathan Vickers
}

The purpose of this case study is to examine possibilities for improving upon hypermedia design in regards to readers and their varied levels of prior knowledge. By creating a hypermedia website that looks at the development of rock climbing at Coopers Rock State Forest, I will explore the possibility of using prompts intended to help prepare readers with low levels of subject (rock climbing) knowledge to understand and make sense of the content. However, at the same time, it is important to allow readers with high levels of subject knowledge to bypass these prompts because of their ability to limit a high knowledge reader's tendency to engage in active processing. This project will provide a model to act as a means of comparison and critique for future hypermedia designs. 
TABLE OF CONTENTS

$\begin{array}{lc}\text { ABSTRACT } & \text { i }\end{array}$

TABLE OF CONTENTS

$\begin{array}{lc}\text { REVIEW OF LITERATURE } & 1\end{array}$

$\begin{array}{lr}\text { METHOD } & 12\end{array}$

$\begin{array}{lr}\text { THE CLIMBING COOPERS PROJECT } & 17\end{array}$

$\begin{array}{lc}\text { DISCUSSION AND CONCLUSION } & 34\end{array}$

REFERENCES 42 
With any new medium or channel there is a period of time needed for adjustment. Both content providers and consumers are going through an obvious transitional period because of these new channels that allow for a multitude of media and interactions to take place. The technologies surrounding the internet have increased and dramatically changed the way content can be presented and consumed. Hypermedia is emerging as a new medium that has the potential to present content more vividly and closer to actual experience than ever before. However, we are still figuring out exactly how to create and consume it.

Hypertext is the method of nonsequential reading and writing where individual chunks, or nodes, of information can be viewed in a variety of arrangements (Borsook, 1992); hypermedia extends hypertext to include the full gamut of media. This goes beyond only text nodes and includes sounds, graphics, animations, videos, music, etc. There have been some great journalism works moving in the direction of hypermedia such as The Dragon Children, a piece about Chinese-Australian students and National Geographic's Photosynth which allows readers to explore virtual images of places around the world. However, many current web stories look identical to their printed counterpart, and journalists don't always take full advantage of the internet's capabilities (Fredin, 1997). This could be because of the difficult, time-consuming process of creating a hypermedia piece or the vast set of skills required to use and integrate a variety of mediums. It has also been pointed out by several researchers that these stories, with their nonlinear nature, can be hard for readers to consume (McDonald \& Stevenson, 1996) for a variety of reasons, including a lack of format familiarity and a lack of domain or subject knowledge (Lowrey, 2009; McNamara, 2001; Lawless, 2007). This project is an attempt to create a potential model for addressing the latter of the two problems. 
This project will be the creation of a hypermedia document (in this case a website made with a WordPress theme) that portrays the climbing community's development and culture surrounding Coopers Rock State Forest. What will make this project different than other hypermedia stories is the inclusion of prompts that will prepare various readers to consume the content effectively. The literature has suggested that experienced rock climbers visiting this website would have the prior knowledge to navigate and make connections between story fragments; however, non-climbers would have a tough time given their limited and potentially incorrect understanding of the community and specifics of the sport. To address this, the site will include an option to view a prompt or prompts that will help to improve a non-climber's prior knowledge. The hope is that they will be better able to navigate and understand the hypermedia document, the complexity of the stories at Coopers Rock, their relations to one another and therefore be able to make their own comparisons to their particular rock climbing experience.

\section{Review of the Literature}

Our brains and experiences are anything but linear. Borsook (1992) believes that the many linear methods of storytelling are inadequate, and nonlinear storytelling methods such as hypermedia are becoming the subject of some debate. Hypermedia, in its many forms and with its many titles, can be found in the research of many fields including journalism, education technology, and man-machine interfacing. This has led to an inconsistency of terms between and within these many fields of study (Geest, 1994). The following is an attempt to cover some of the main concepts and vocabulary surrounding hypermedia as a journalistic format for telling stories or sharing information. It will discuss its benefits, downfalls and the research surrounding how audiences have engaged with these types of media. By looking specifically at education 
technology research, it has been proposed that readers of hypermedia formats may learn in different and potentially deeper ways (Boorsook, 1992; Jacobson \& Spiro, 1995). Cognitive flexibility theory (CFT) will serve as a framework for this research, which will explore audience engagement with hypermedia and will ultimately create a model for hypermedia storytelling. Its design will attempt to address several problems in regard to audiences' variety of experiences and different understandings of domain and content formats. It will not only act as an example of how journalists and other communicators can prompt an audience but also provide design considerations for effective authoring of hypermedia stories that engage a diverse audience.

\section{Hypermedia}

Much like Borsook’s (1992) Hypertext definition, hypermedia is a nonlinear format that establishes relationships between nodes, allowing for a more meaningful reader experience and extending the definition to a variety of mediums (Ayerman, 1993). While paper documents are, in principle, linear; hypermedia is nonlinear and non-sequential. It is important to note that hypermedia, often found as websites, is not the only nonlinear format and is not necessarily a website or even on a computer (Borsook, 1992). Often used interchangeably with the word hypertext, the term hypermedia includes the full range of modes such as sound, graphics, animation, video and text, whereas the term hypertext is limited to only text. One might refer to hypermedia as multimedia hypertext (Borsook, 1992).

Hypermedia's strength may be found in its web-like structure of connected bits of information. These bits, known as nodes, are connected via hyperlinks, which provide information about the relationships between various nodes and allow readers to navigate the content in a variety of ways (Geest, 1994). Hypermedia focuses on the connections between content parts (nodes) as much if not more than on the content itself. This allows for a more 
accurate depiction of complex subjects (Borsook, 1992; Ayerman, 1993). It has been suggested as a format most suited for providing instruction in complex and ill-structured domains. Lowrey (2009) defines an ill-structured domain as, “a knowledge area that varies substantially across contexts in which knowledge is to be applied, which does not have a consistency of terminology across instances, and which defies efforts to pre-specify conditions for knowledge use. These illstructures domains could also be referred to as complex case specific problems or stories (Jacobson and Spiro, 1995). These ill-structured domains and complex stories could include the entire gamut of complex public issues covered in our daily news (Lowrey, 2009). These formats may work because of the way we organize information. Theorists have discussed hypermedia's parallels to the human brain and the ways we construct meaning from a sea of interconnected experiences (Borsook, 1992).

Unfortunately, the current research regarding hypermedia is inconsistent at best (Salmeron, 2005). This may explain why many journalists have yet to embrace this new format in their storytelling. For example, Massey (2004) examined 38 Web newspapers, showing that nonlinear storytelling for news was rare. While some studies support the benefits of incorporating hypermedia formats (Jacobson \& Spiro, 1995), others have revealed the shortcomings of a hypermedia approach such as disorientation (McDonald \& Stevenson, 1996; Stanton, 2009). Its effectiveness may be inhibited by an audience's lack of familiarity with the format, the subject matter itself, or a combination of the two. In any case hypermedia is a format that is becoming more and more prevalent as technology develops (Borsook, 1992). Recent research points to ways hypermedia can be better implemented, and methods for limiting its pitfalls and highlight its strengths are emerging (Strobel, 2008; McEneaney 2009; McNamara, 2005; Lawless, 2007). 
McNamara (2005) started by identifying the possible relationships between a readers prior knowledge and the cohesion of a hypertext. He discusses the how to help low-knowledge and domain novice readers get the most out of hypermedia and the fact that high-knowledge readers and domain experts don't require as much cohesion. Later, it was shown that prompts or pre-reading activities could be used to change this prior-knowledge and stance of a reader to allow for a greater knowledge transfer, a deeper understanding and more rewarding experience of a give hypertext (Lawless, 2007; McEneaney, 2009).

There is a great deal of inconsistency with the terminology describing the hypermedia format. Words such as multimedia, hypertext, hypermedia and Cognitive Flexibility Theory format are often used interchangeably, making consistency a struggle. This proposal and project will use the term hypermedia (and, where more applicable, Cognitive Flexibility Theory format) to describe this format of linking a mosaic of nodes to create a non-linear story. The benefit of this term over hypertext is that it includes the entire gamut of multimodal forms such as video, photo, text and so on whereas the term hypertext is limited to only linking through text (Borsook, 1992). Because of this ability to use various modes, hypermedia creates a richer learning environment. It can integrate a multimodal set of nodes or content segments into a nonlinear format allowing a hypermedia text to be more comprehensive and provide a multitude of perspectives and cases that a reader can construct knowledge from (Ayerman, 1993; Borsook, 1992).

Hypermedia, which is particularly valuable for complex or multiple perspective storytelling, can be used as a means to express unlimited stories, ideas and concepts. There is also an unlimited amount of ways to connect and display this content. These can be simple designs that link and organize three videos from different perspective such as in the New York 
Times piece In Economic Vise, Pontiac Struggles or elaborate web spaces that allow you to virtually explore actual museums from around the world such as in Google's Art Project. Various levels of linearity can exist and some almost seem more like a videogame rather than a story because readers are expected to take part in control the narrative by making decisions or moving through a virtual space. The Block is a great example where the user or reader can explore “The Block” and its stories by virtually walking through its streets, while Bear 71 allows a user to explore the world of tagged animals. These stories and others like them are great hypermedia examples that have great merit according to Cognitive Flexibility Theory (CFT).

\section{Cognitive Flexibility Theory}

Originally associated with learning psychology, Cognitive Flexibility Theory (CFT) attempts to explain how the brain makes sense of complex case-based information and has begun to emerge in the field of journalism and communication (Lowrey \& Kim, 2009; Borsook, 1992; Lowrey \& Choi, 2006; Strobel, 2008; Jacobson \& Spiro, 1995). With roots in constructivism, where learning is an active process of building new knowledge, CFT assumes knowledge is organized into schemas within the reader's long-term memory (Lowrey, 2009). Within cognitive flexibility theory, hypermedia is created by both the author and reader (Geest, 1994). The author defines the content and linking, while the reader selects elements and determines the sequence of viewing the nodes.

CFT is usually applied to advanced learning within ill-structured knowledge domains where consistent and simplistic designs are unable to convey the complexity of the content. This approach has been suggested as a way to tackle topics such as economics, medicine and engineering. (Lowrey, 2006; 2009; Strobel, 1995; Borsook, 1992). These domains of learning and many others are often difficult to present with absolutes and can be victims of 
oversimplification within a linear format. Many authors have suggested the nonlinear structure of hypermedia (often in the form of websites) is a CFT format because of its ability to associate many case-based examples and a variety of conceptual perspectives (Lowrey, 2009; 2006; Borsook, 1992; Jacobson \& Spiro, 1995; Strobel, 2007). Despite the theoretical belief in hypermedia’s potential for learning, the research results in this area have been considerably inconsistent (Lowrey, 2009). Some studies show many benefits of hypermedia in regards to knowledge transfer and efficiency (Jacobson \& Spiro, 1995; Lowrey, 2009) and others show no benefit to using this format over linear content (Lowrey, 2006). This could be due to variety of factors including but limited to subject matter, a readers familiarity with the format or the navigation design. Some studies point to its many downfalls, such as disorientation and literacy problems, saying they outweigh its benefits (Borsook, 1992; McDonald \& Stevenson, 1996).

\section{Empirical Findings of Hypermedia Effectiveness}

Early theoretical literature suggested that hypermedia, or the multimodal and non-linear delivery of information, would be more beneficial for all learning due to its ability to make connections and semantic structure (Borsook, 1992; Ayerman, 1993). Unfortunately, later studies failed to confirm this proposal (McDonald \& Stevenson, 1996; Stanton, 2009). McDonald and Stevenson (1996) suggest that this was due to reader disorientation and navigation problems. However, conflicting research does exist, such as a study of news radio that showed that test subjects performed better with nonlinear news listening in both recall and comprehension (Mesbah, 2006). Much of this inconsistency could be attributed the recent emergence of hypermedia, its diverse nature and the fact that its study crosses several fields. Geest (1994) explained that many studies have only looked at novice hypermedia users, using small hyperdocuments and covering domains that test subjects have little experience with. 
Important distinctions are emerging in regards to hypermedia and is has been suggested that there are certain types of learning that hypermedia may be more appropriate for. Jacobson and Spiro (1995) showed a relationship between hypertext and the types of knowledge a reader receives from it. Their study showed that readers of a minimal hypertext/drill presentation (nonhypermedia) performed better on memory-oriented factual knowledge items than those reading a thematic crisscrossing hypertext (hypermedia). However, when subjects were tested for higher order cognitive skills with a knowledge transfer problem-solving essay, those who were in the thematic crisscrossing group (hypermedia) outperformed those in the minimal hypertext/drill group.

Despite the initial research, recent studies have suggested not only that hypertext improves higher-level learning, but that understanding certain reader attributes such as stance, motivation and prior knowledge may drastically influence the success of hypermedia (Jacobson \& Spiro, 1995; Tremayne, 2003; McEneaney, 2009; Salmeron, 2005; McNamara \& Shapiro, 2005; Lawless, 2007; Lowrey, 2009). These reader attributes and many other distinctions must be explored before casting hypermedia to the side.

\section{Prior Knowledge, Cohesion and Prompting}

Many specifics of hypermedia, such as prior knowledge, cohesion and prompting, are still being explored because of their effects on a reader's hypermedia experience. Lowrey (2006) showed that readers are less clear about CFT formats such as hypermedia, suggesting a significant interaction between prior knowledge and a reader's interactions with hypermedia formats. Prior knowledge seems to have as much of an effect on a reader as the format, which may explain many of the inconsistencies in much of the earlier research of hypermedia. Lowrey (2009) used a CFT format in the context of journalism. While he found little evidence that the 
CFT formats benefit readers other than an increase in reader enjoyment, he did find correlations between prior knowledge and a reader's opinions of subject and navigation patterns. Those with prior knowledge were more likely to have more moderate views on subject matter, did more scanning of the content and engaged the content in a more nonlinear way.

This importance of prior knowledge has since been applied to CFT and hypermedia more directly (Lowery, 2009; Lawless, 1997, 2007; McNamara, 2001; Salmeron, 2006). It is becoming more and more apparent that the benefits of CFT and hypermedia formats are dependent on learner characteristics such as prior knowledge, interest, stance and self-efficacy (Lawless, 1997).

It has been shown that prereading activities can increase a reader’s prior knowledge and help with nonlinear engagement (Lawless, 2007). Others have shown that a reader's stance, the purpose or general orientation of a reader, is also very important and can be influenced by prompts (McEneaney, 2009). Reader characteristics such as these can drastically change a reader’s experience and learning with CFT formats and hypermedia; consequently, a lot of research seems to be moving in this direction.

Researchers are now exploring these characteristics and how they interact with the structures of CFT formats and hypermedia. A reader's prior knowledge can have a distinct relationship with the 'cohesion' of a story. Cohesion being the information contained in separate nodes (text, video, graphic etc.) and their connection to each other within both the hypertext and the reader's mental model of the hypertext (McNamara, 2005). McNamara (2001) showed an interaction between prior knowledge and the cohesion of traditional texts before Salmeron (2006) went on to show similar relationships with hypermedia texts. His study showed that lowknowledge readers do not learn effectively from low-cohesion designs, and further studies by 
McNamara (2005) have suggested that high-knowledge readers can benefit from low-cohesion texts.

McNamara (2005) discussed the application of various levels of coherence in regards to hypermedia. He suggested that low-cohesion texts, such as hypermedia, can be more beneficial for learning because the reader is required to slow down and engage with the content in order to make sense of it. This process can give a knowledgeable reader a deeper and richer learning experience. The low-cohesion text's ability to slow knowledge acquisition may help with longterm retention and intra-task transfer, while a high-cohesion text might inhibit active processing. However, not all readers have the level of prior knowledge to engage and benefit from these lowcohesion tests. McNamara (2005) also described solutions to the problem of serving a diverse population of readers with guidelines for hypermedia design aimed to accommodate individuals’ knowledge and learning style.

It has become clearer that hypermedia isn’t always better than linear text, but must be leveraged effectively in order to reap its benefits. If the target audience is not considered and their objectives and perspectives kept in mind, a hypermedia text can simply confuse them. Much like Lawless (2007), who was able to frontload a reader experience with a pre-reading to increase prior knowledge, McEneaney (2009) designed prompts to induce a specific stance from a reader. He first showed that he could induce a specific stance from a reader with a prompt before coming to conclusions about those specific stances and how they affect a readers’ interaction with hypermedia content. He was able to encourage a more aesthetic stance, where a reader focuses primarily upon the experience lived through during the reading as opposed to an efferent stance where a reader is more concerned with what information they can walk away 
with. Through recall and essay writing tasks, he was able to show that those prompted to take a more aesthetic stance attained a higher level of understanding.

Hypermedia research is beginning to make sense of this complex format. Its benefit in multi-faceted storytelling is becoming clearer (Tremayne, 2003) and much of the research has started to reveal what the theory has suggested all along. Hypermedia seems to be a format that benefits knowledge transfer as opposed to memory (Jacobson \& Spiro, 1995). The research has also illuminated the fact but that high knowledge readers can benefit from hypermedia's lack of cohesion and that high levels of cohesion can even inhibit their active processing (McNamara, 2005) while low knowledge readers struggle with the limited cohesion of many hypermedia documents (McNamara, 2005; Salmeron, 2006). While these characteristics make hypermedia a great option for telling stories to high-knowledge readers such as distinct communities and experts, it is also important to explore how hypermedia can be designed to address the discrepancies within a wide range of readers.

The recent research regarding hypermedia design suggests the consideration of prior knowledge and stance when developing hypermedia stories (Lowrey, 2009; Lawless, 2007; McEneaney, 2009). This project will explore possible real world solutions through the creation of an original hypermedia story that depicts the rock climbing community surrounding the development of Coopers Rock State Forest. Its design considerations will be based on literature in hopes of creating an example of how or how not to correct for these audience and potentially other audience variables. 


\section{Method}

The project was the creation of a hypermedia website, using a preexisting WordPress theme that explores rock climbing at Coopers Rock State Forest. Located outside of Morgantown, West Virginia, Coopers Rock has been the site of rock climbing for decades. This story illuminated the many people, stories, issues and institutions that have shaped the climbing development and climbing culture there, many of which are applicable and relatable to other rock climbing and recreation communities. It is intended to serve as an example of how to create a hypermedia story for complex stories and accommodate readers of various knowledge levels when telling a story so specific to a community.

\section{Hypermedia Design}

Lowrey (2009) says that one of the biggest problems journalists face when considering a CFT format is whether a story is a good fit for it. This is important for content producers not only because of the great deal of work that these types of stories require, but also because of the nature of the format. It has been suggested that complex stories lend themselves to CFT formats such as hypermedia websites, but it is important that the audience has some knowledge of the subject (Lowrey, 2009; Borsook, 1992). I expect my model will be more easily understood by readers who are part of the rock climbing community or have prior knowledge with similar recreation activities; however, I made design considerations to make this story accessible to more readers. My hope is that all readers can walk away with an understanding of the subject and its many dimensions.

While hypermedia can be used to tell any story it is most useful for certain types of stories. It is most beneficial when the story can be told with a collection of interconnected elements (Borsook, 1992; Geest, 1994). I used many small stories and story fragments (nodes) 
relating to rock climbing at Coopers Rock to create a hypermedia text that will tell the broader story of climbing at Coopers Rock. The story explored the different people, places and influences that not only make Coopers Rock what it is today, but have influenced rock climbing as a whole. According to CFT, these individual stories are real-world cases and perspectives that readers can associate with one another and with their own opinions and experiences (Lowrey, 2009). The literature suggests this allows for a greater level of knowledge transfer (Jacobson \& Spiro, 1995).

As in many hypermedia texts, the individual nodes are presented in a variety of media, including text, video, photo and graphics. In many cases multiple modes are used together with one another and the navigation of the website. Many of the more complex concepts are presented with some text and a large video component much like a short web documentary. Other concepts are presented through still images, info graphics and text (bios, description, captions etc.) depending on the complexity and content being presented. This use of multiple mediums can be seen in The Dragon Children, a hypermedia piece uses different mediums to tell different aspects of a multidimensional story about Chinese students succeeding in the Australian school system.

The Navigator WordPress theme, while designed for travel blogging, made for a good structure that allowed for many characteristics important to hypermedia non-linear and spatial navigation and storytelling (Mancini \& Scott, 2006). The theme allowed me to link nodes in a variety of ways and provides map-based and traditional navigation options allowing for a third characteristic the literature considers important to hypermedia: non-linearity (Mancini \& Scott, 2006; Borsook, 1992; Geest, 1994; Ayersman 1993; Strobel, 2007). This non-linear story can be 
navigated in a variety of ways, which will allow different readers to explore the nodes in their own way and pursue the things that interest them.

\section{Audience Considerations}

While good hypermedia's navigation is important, the success of it is just as influenced by its readers. The usability of a site is increased if reader variables are accounted for (McEneaney, 2009). While there are a variety of other audience variables, I believe the discrepancy between knowledge levels to be the greatest hurdle, followed by format familiarity and personal experiences and opinions.

Given the nature of rock climbing (various types, technical info and jargon etc.), this story will have two drastically different types of readers: climbers and non-climbers. Climbers, with their experience and familiarity with terms and situations related to climbing, would be considered to have a high level of knowledge while non-climbers would fall at the other end of the spectrum. According to the literature this high level of prior knowledge will allow the climbers to not only understand the content, but more easily incorporate their own experiences with the text (McNamara, 2001). In fact, it has been shown that those with greater prior knowledge of the given subject or domain are more likely to use non-linear navigation, spend more time with content and view more information across modalities (Lawless, 2007). Others have even suggested that highly cohesive texts which explicitly connect concepts and content nodes may limit a knowledgeable reader’s active processing (McNamara, 2005; Kalyuga, 2007). McNamara (2005) refers to this as the reverse-cohesion effect, while Kalyuga (2007) uses the term expertise reversal effect.

While some research points to the benefits high-knowledge readers get from lowcohesion texts, even more evidence points to the limiting effect a low-cohesion text has for the 
low-knowledge reader (McNamara, 2001, 2005; Salmeron, 2006; Lawless, 2007). It has been shown that readers of nonlinear texts who lack domain knowledge can become disoriented and struggle with navigation (McDonald \& Stevenson, 1996). These readers are more dependent on coherent navigation such as menus (Lawless, 2007). Fortunately, possible solutions to this problem are being developed and studied (McEneaney, 2009; Lawless, 2007). Attempts to add optional content and explanation to stories can be seen with the use of links during other content such as the more info box found during the videos in Quenching Las Vegas’ Thirst.

Within the education field, many have had aspirations for learner-tailored instruction that could account for differences in a reader's prior knowledge, but ultimately it has yet to be fully realized (Kalyuga, 2007). Given the expertise reversal effect and empirical evidence that suggests low-knowledge readers struggle with low cohesion texts, a hypermedia website that is intended for a diverse audience must account for this discrepancy. Both Lawless (2007) and McEneaney (2009) have looked at the ability different prompts and pre-reading activities can have on a readers ability to engage with hypermedia. It has been shown that a reader's purpose or general orientation (stance) can be influenced by short text prompts and that encouraging a more aesthetic stance can lead to better use of built in navigation and a better understanding of hypermedia content (McEneaney, 2009). Prompting can be seen when a reader enters sites such as Bear 71 or The Block where the reader is first prepared for the type of content they are going to receive and receives an overview of the basic story information. It has also been shown that administering pre-reading activities designed to increase a reader's prior knowledge can encourage nonlinear reading, viewing across modalities, longer times spent with content and an increase in scores on post knowledge assessments (Lawless, 2007). My design includes prompts to both prepare readers for appropriate engagement with my hypermedia text and to increase the 
prior knowledge of low knowledge readers. I hope Climbing Coopers maintains an appropriate low cohesion design to encourage active processing and the incorporation of prior knowledge, while allowing for a diverse audience. 


\section{The Climbing Coopers Project}

The shooting, editing and web design for the Coopers Rock Project took a little less than two years, from January 2012 until July 2013. The first step, which included shooting and editing individual content fragments, was the primary focus of the first year of my project. I collected interviews and constructed various narrative threads that would together tell the story of climbing at Coopers Rock. During this time I was able to collect various artifacts, such as a page from an out-of-print climbing guidebook (see figure 1), that were later scanned into digital copies and included as content fragments in the website.

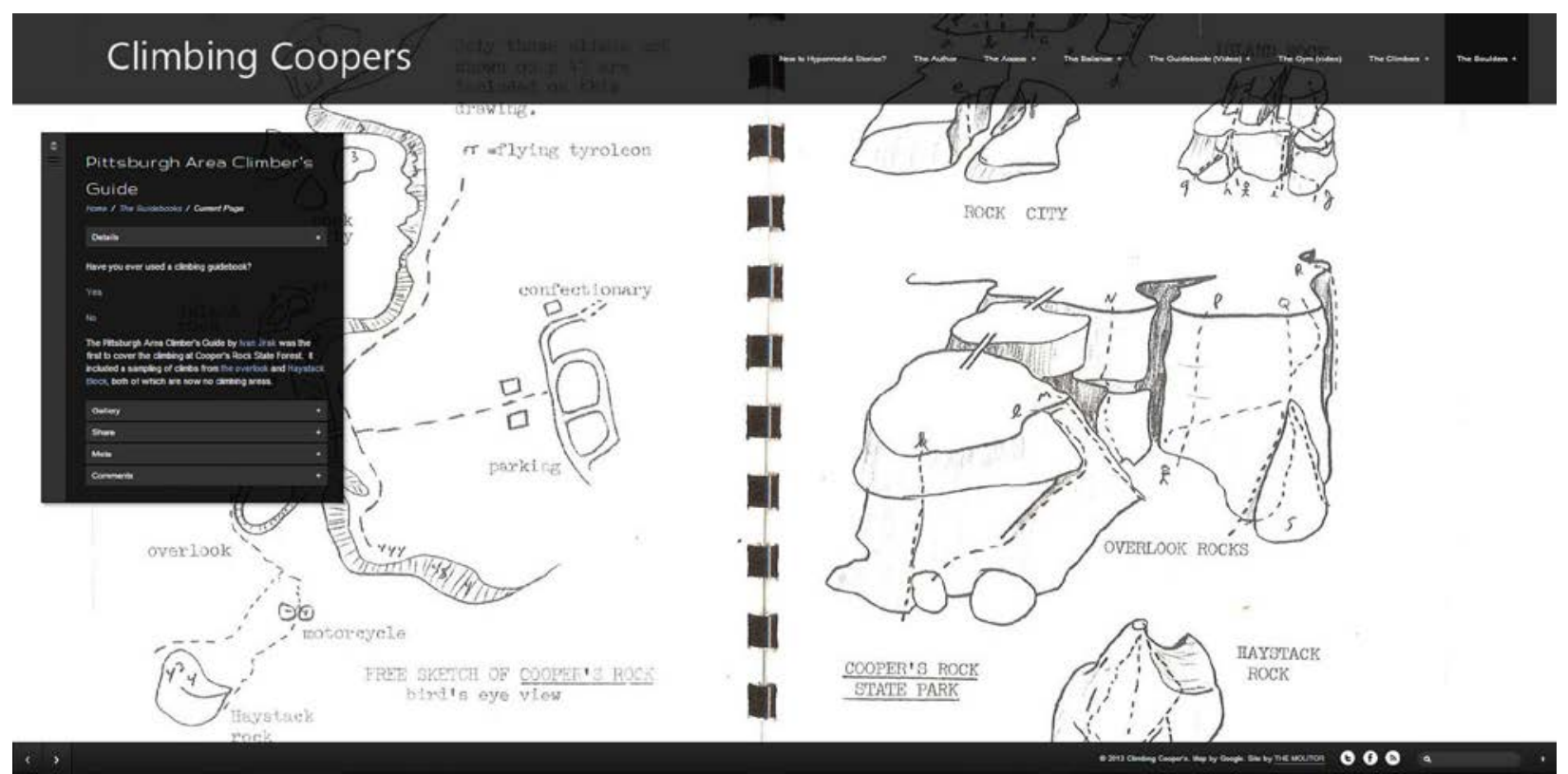

Figure 1. A page on the Climbing Coopers website that contains info and images about the out-of-print guidebook Pittsburgh Area Climber's Guide by Ivan Jirak.

Climbing Coopers (climbingcoopers.com) is the story of climbing at Coopers Rock State Forest, which is located outside of Morgantown, West Virginia. It is a collection of individual story fragments that work together to express the various components and perspectives that can exist in a small climbing community. The website's story fragments are organized with a 
dropdown menu found at the top of the page, and the pages also appear as pins in the map of Coopers Rock State Forest found in the center of the page.

\section{The Website Structure}

While working on the individual video segments, I started deciding on the structure of the website that would house the project. I chose to use a Wordpress site because of my familiarity with it, having worked with it before. Since I was collecting content while deciding on a theme, the two considerations somewhat influenced one another. I wanted a theme that allowed the navigation or interface to help illustrate the story in some way. My original idea before collecting and editing all of the content led me to think a map-based interface might be useful given the geographic nature to rock climbing. Most Wordpress themes seem to be variations on the same basic navigation in that a horizontal bar at the top contains links and potentially dropdown menus with more links. However, I was able to find a map-based theme called the Navigator. The Navigator theme was better suited to my story that had such strong ties to places and objects (see figure 2).

Google map and menu navigation. The theme’s geographic navigation was an embedded Google map with pins that contain information and links to other pages and posts. I saw the story fragments as being confined to specific locations that could be navigated via a Google map integrated into the theme. Some of the information was definitely geographic in nature, such as information about specific climbing areas or the videos about access issues with particular rock features, but others were better linked by concepts related to Coopers Rock. These would include stories about well-known area climbers and publications such as guidebooks which didn’t necessarily have a specific geographic location affiliated with it other than its tie to Coopers Rock as a whole. As I edited my content and integrated it into the website, 
I found myself using the Google map navigation (see figure 2) less than expected, opting for the more traditional menu navigation (see figure 3). However, I think having all of the content and editing planned before choosing an interface or structure would be of great benefit because an author can then weigh the benefits and limitations of certain structures given the particular content and story components they are working with.

Stories and content fragments can be found through both the menu and map navigation on the Climbing Coopers website. Once a reader has started with one of these options of navigation they can switch to using hyperlinks for navigation or continue to use the navigation they originally choose. However, some pages do not allow a user the ability to use map navigation because I instead decided to use a storytelling image in its place. When geographic context was beneficial the map navigation was kept. This can be seen in the Boulders section where the map navigation allows a reader to select different locations on a map to explore further. This diversity of options allow for different readers to have a variety of experiences based on not only what content fragments they happen to see, but also in how they navigate to those content pieces.

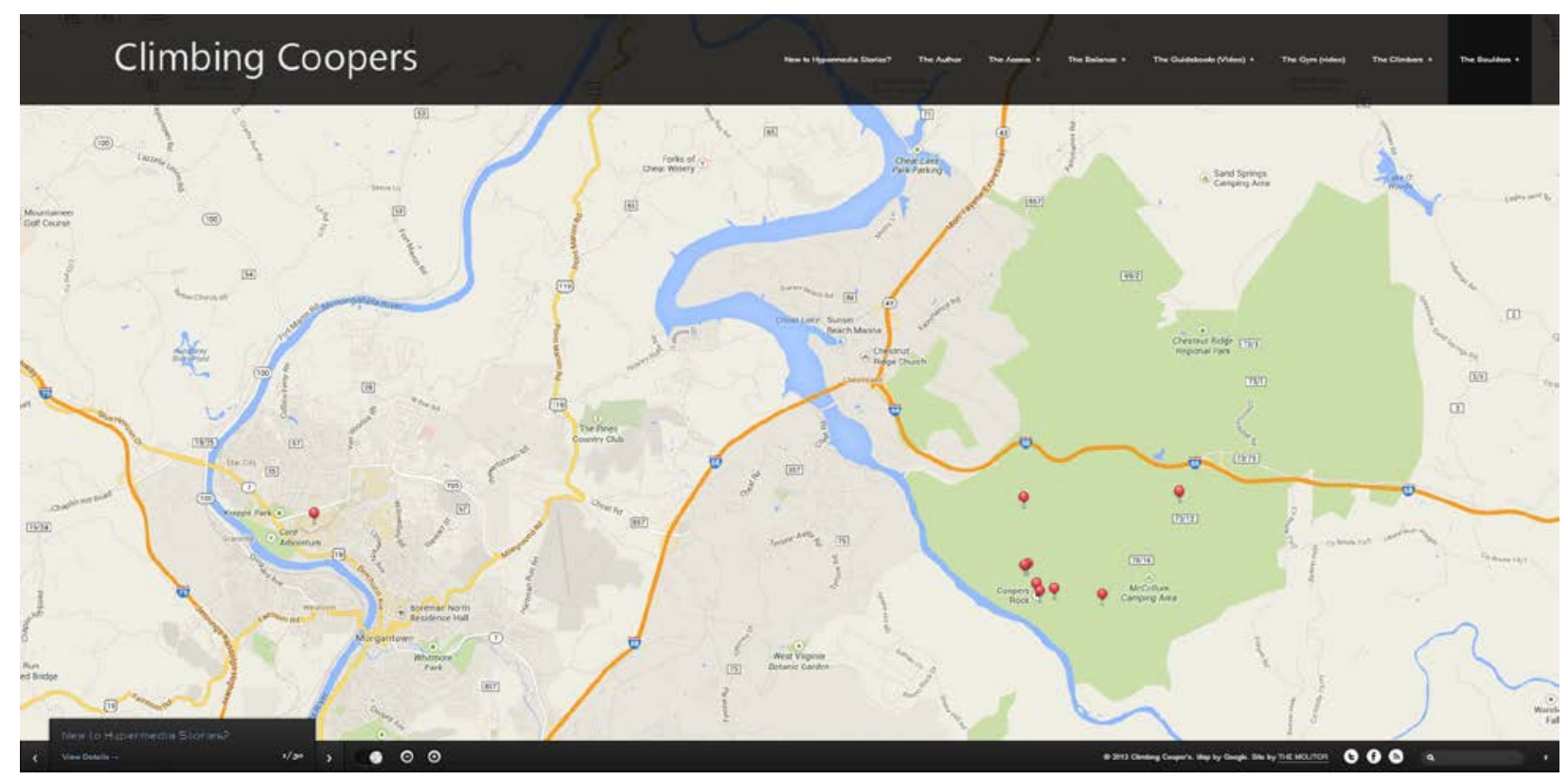


Figure 2. This is the home page of Climbing Coopers. The content can be navigated via the menus at the top of the page or the interactive Google map.

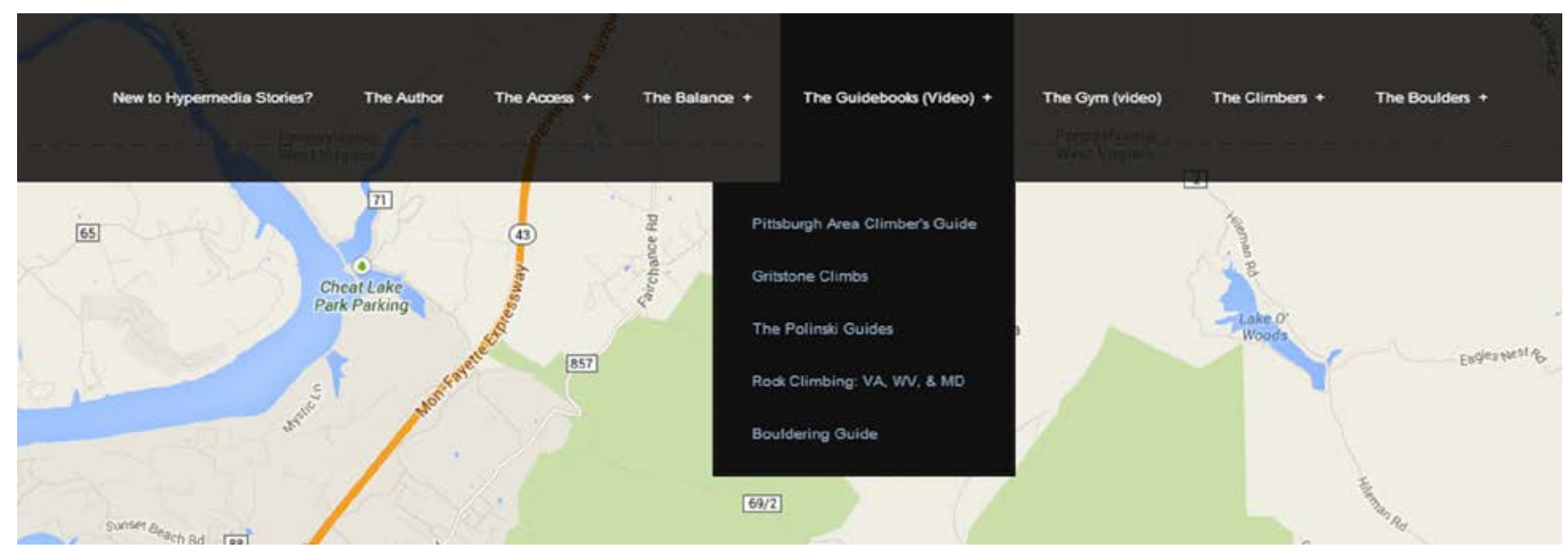

Figure 3. A close up of the navigation on the website. This shows the dropdown menus that were often used to distinguish differ content piece under a specific category of topic.

Since not every page needed to have its own map navigation, maps that provided no real information or contextual connection to the content or story were replaced with more telling images. Various images, like the backgrounds I used for many of the posts and some of the pages, provided more information about the relationship between content pieces and allowed for another medium within a page or post. Overriding the Navigator theme's maps took some time, but with the help of Alex Yohn, a coder for WVU, I was able to make image backgrounds override the map feature of these pages while keeping their geographic information. This information was required for all posts to allow the map function to work and maintain their map presence on other maps in the site. Alex continued to help from time to time with some of the coding and site customizations, including helping me to make the home page map display the Morgantown area as opposed to Paris.

The Access posts are a great example of a situation where, even with the stories' geographic relationships, the Google map didn’t give a reader much detail. The old aerial photo (see figure 4) used instead of the Google Map gave the same basic geographic information but 
allowed a reader to see the size of the actual rock features in relation to each other and the landscape as opposed to simply where they are. The old aerial photo of the two areas gives a reader much more information and is another artifact that I preferred because it tells its own story. I used some other old photographs and artifacts to show examples, portray concepts and evoke emotion throughout the site. These old photos and artifacts can be seen throughout the Climbing Coopers website (see figures 4 and 5).

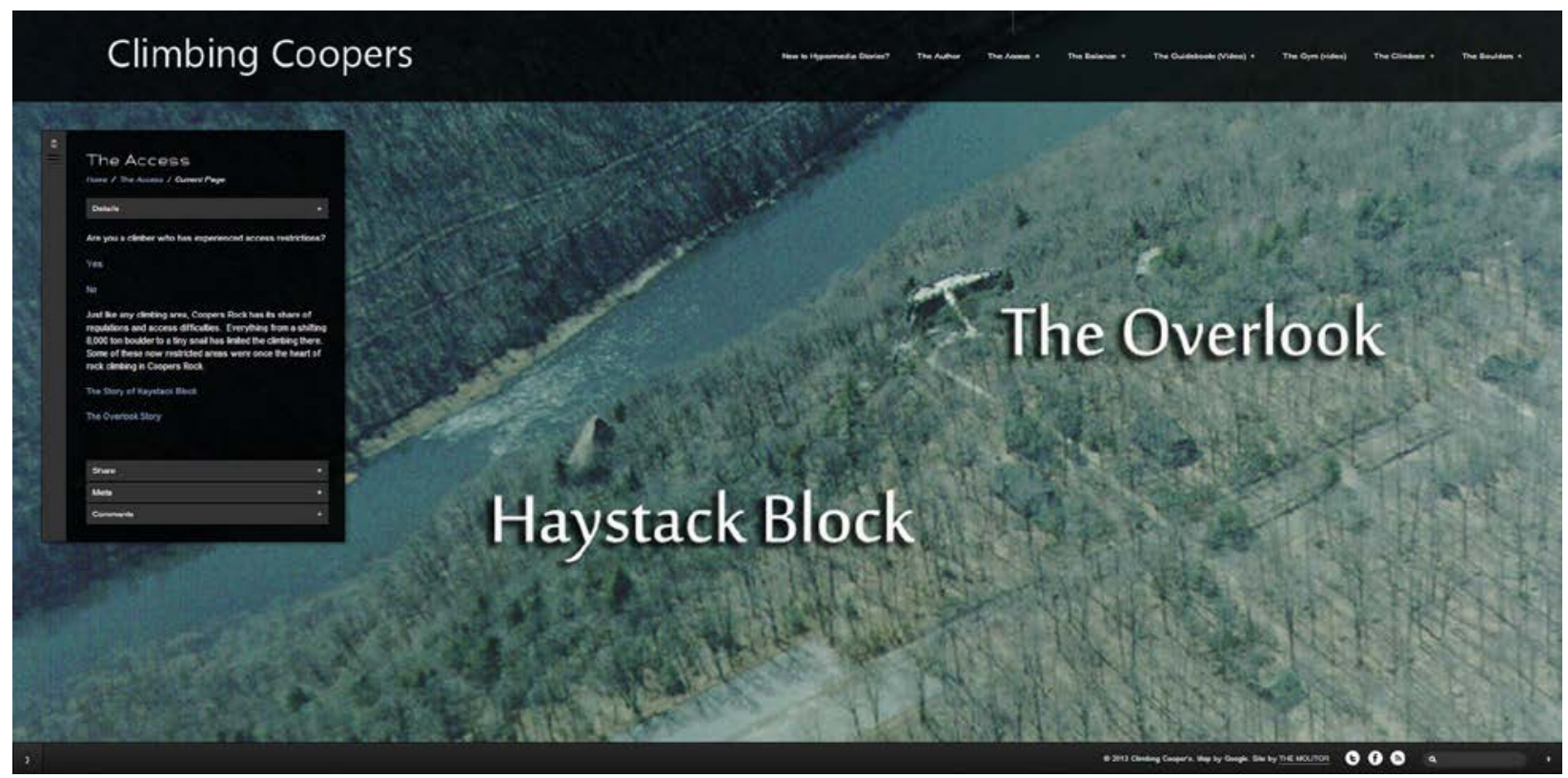

Figure 4. This page acts as an introduction to the concept of problems with climbing access. At first this page was a Google map with two pins, but this old photograph gave more detail and allowed for a reader to see the size of these rock features in relation to the landscape. 


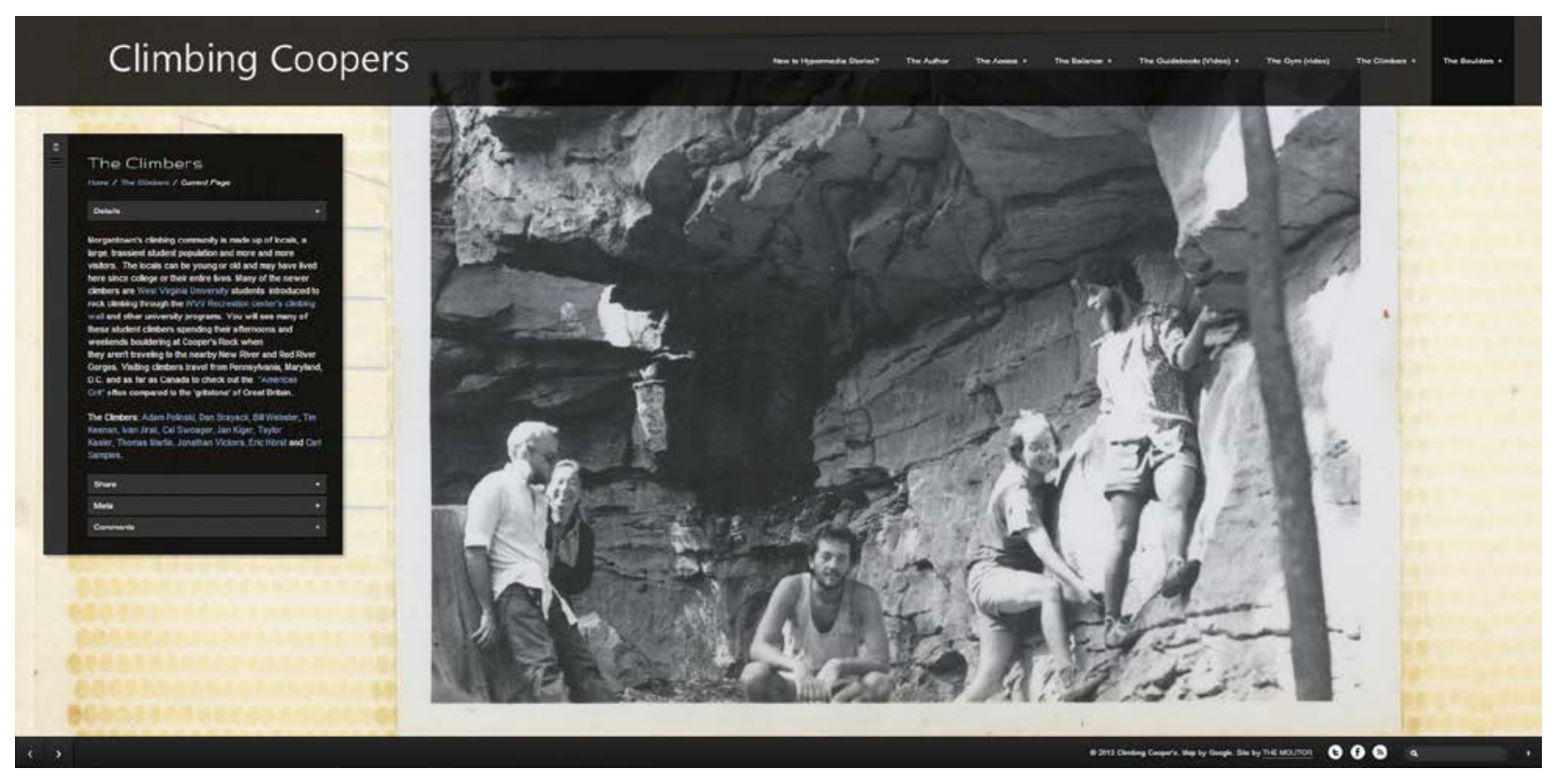

Figure 5. This is a page that introduces the climbing community surrounding Coopers Rock State Forest. The background image is a throwback image from Adam Polinski's collection of photos. You can see the many hyperlinks that link to other story fragments in the text box on the left.

Hyperlinks and navigation. The third navigational component was in the form of hyperlinks. In hopes that every visitor could have a somewhat different experience with the content, I added a lot of hyperlinks throughout the website (See figure 6 and 7). My expectation was that readers would start exploring the site with either the menu or the map navigations, but that they might use hyperlinks as well once they got started. The conceptual linking to related content through hyperlinks would allow them to jump from one story or topic to another related section of the website or even external content. This allows for a variety of options as the reader moves though the stories in the order of their choosing. These links included links to other content pieces within the Climbing Coopers website as well as additional pages of related content from the Internet. 

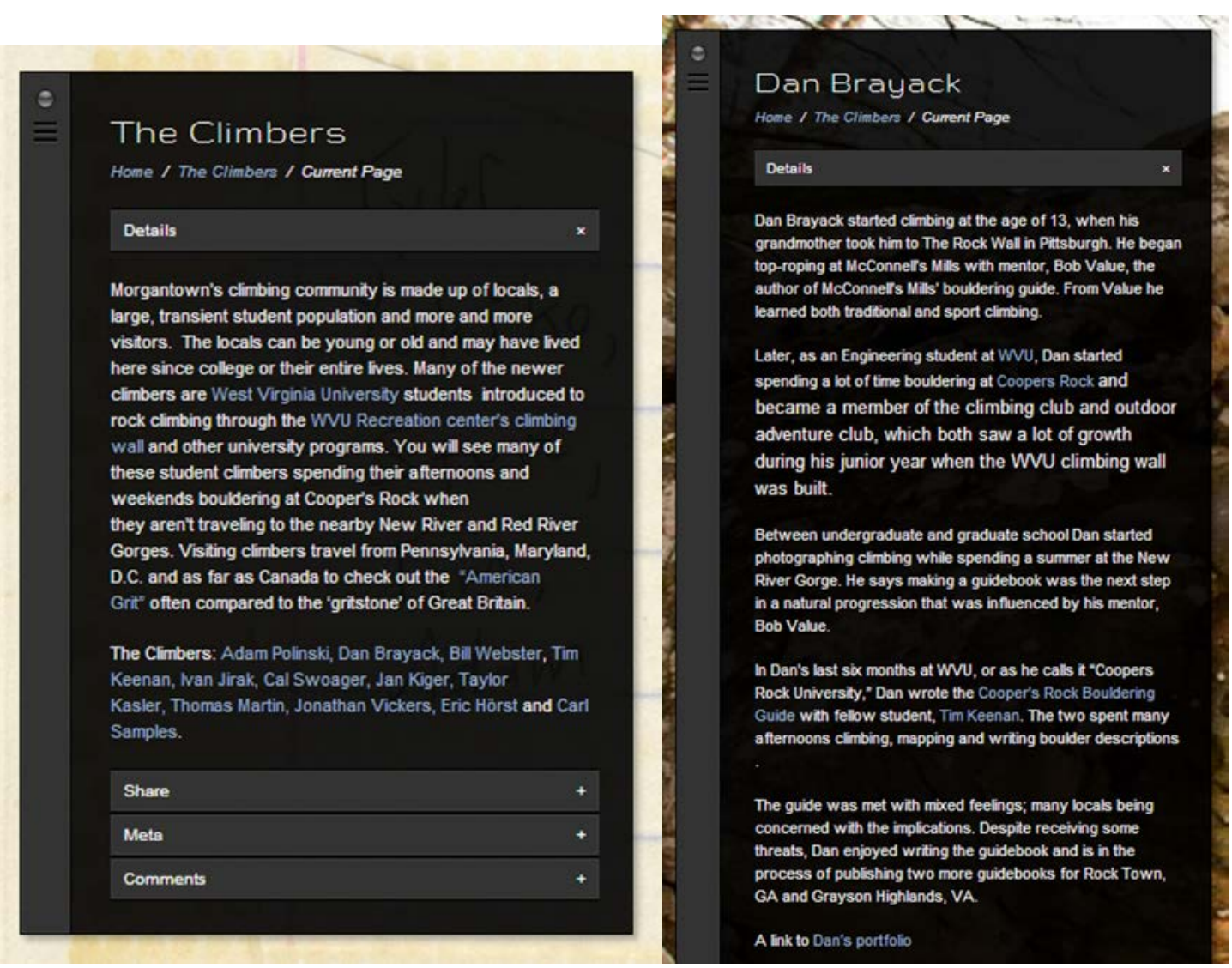

Figures 6 and 7. This shows two examples of the use of hyperlinks in the text of the website. The left image (Figure 6) is from the page introducing the climbing community as a whole and the right image (figure 7) is an excerpt from the Dan Brayack profile page.

I ended up with a dynamic combination of navigational options. Readers can navigate using a traditional menu of dropdowns organized conceptually, surf content or explore the areas of Coopers Rock geographically, or simply jump from content fragment to content fragment using the hyperlinks. While I may have strayed away from using a theme completely designed around the Google maps, I do think the geographic navigation adds information and context for climbers who visit Cooper Rock and allows for more reader variability.

As with most Wordpress themes there are some limitations to the Navigator theme that helped to dictate the ways content can be incorporated into each page or post. The goal with the Climbing Coopers website was to be able to incorporate several mediums into each content 
segment or at the very least be able to connect a variety of individual pieces together through the navigation. Without making any changes to any code, the pages and posts of the Navigator theme can contain text, photo galleries and a map. The text fields of these posts and pages can contain embedded content such as videos. Most Wordpress themes allow for these types of content with the map integration being an exception.

\section{The Navigation Menu}

At the top of the page the menu is a row of buttons that link to content segments and provide categories for organizing other content. These buttons link to information about hypermedia, the author, the access, the balance, the guidebooks, the gym, the climbers and the boulders. These topics are meant to represent the major components that make up the local climbing community and the sport of rock climbing in Coopers Rock State Forest. Each topic has a page dedicated to explaining the topic and many of the topics have dropdowns with links to specific stories or information.

The Access page has two different stories pertaining to access issues. "Access issues” being the closures of areas previously open to climbing. This can be for a variety of reasons, but is often related to environmental impact or liability concerns. The Access page itself is an overview of the access issues in Coopers Rock State Forest. The page contains links to more specific stories about The Haystack Boulder and The Overlook. These two climbing features or situations have their own story pages that cover the specifics of their closures.

The Guidebooks topic contains a major story component on its page, but also links to pages that explore the different climbing guides that have been written about Coopers Rock. The primary story is about the balance to be struck between publicizing local climbing areas and protecting the ecology of an area. The pages highlighting the actual bouldering areas have 
descriptions and images from the guidebooks themselves, but they are not designed to act as a guidebook. For more information about access the actual boulders a climber would be better served by a published guidebook or by using Mountain Project (online worldwide guidebook).

The Gym page is a stand-alone story about one student's take on indoor climbing versus outdoor climbing and contains some explanatory text and images of the climbing facility at West Virginia University. Though it is all contained in one page this component has a lot of content and is an important component of the climbing community around Morgantown, WV. The student population is one of the largest user groups at Coopers Rock State Forest and those rock climbing almost always start at the Recreation Center at West Virginia University.

The Climbers topic is a set of climbing profiles. This can be explored by itself, but is most beneficial for linking purposes with the other story components. Climbers who are more familiar with the local community can read without extra info, but those who may not know a specific local climber can link to these profiles for more information. Those chosen to have profiles or bios were chosen because they are active in the local climbing community through activism and impact mitigation or they were influential developers of the climbing in the area. Some bios were added simply because the individuals were mentioned enough in other stories to merit the inclusion of the additional context.

Since climbing rocks is what it is all about, I had to include a component highlighting the bouldering found at Coopers Rock. The Bouldering pages are somewhat like a guide to the bouldering, but they only contain brief descriptions and some photos as opposed to topographic maps and diagrams like you might see in an actual guidebook (See figure 8). I chose to do this because my intent was not to offer that service at the expense of harming local guidebook sales or at the ecological expense of popularizing too many new areas. I did publicize new information 
about what is known as the County Line Boulders, a non-published area that I felt was appropriate to publicize given its climbing density and proximity to the road.

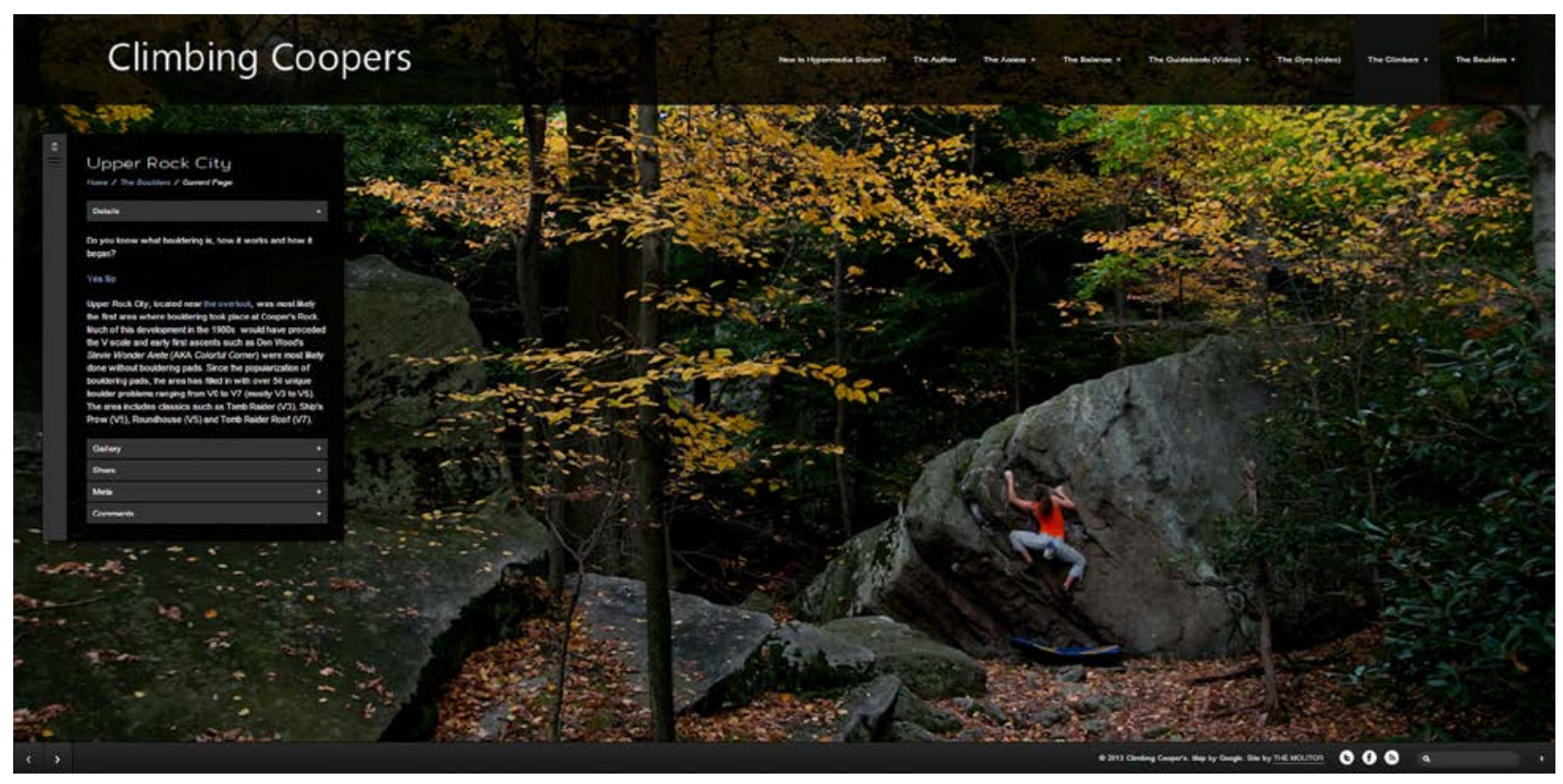

Figure 8. An example of a page describing Upper Rock City, one of the specific bouldering areas found at Coopers Rock State Forest.

\section{Content Creation}

The video components to the Climbing Coopers website were edited using Adobe Premiere and embedded into the text fields of the appropriate post or page (see figure 9). For the most part the videos were used as standalone pieces that were included on their own page/post with only minimal text and maybe a related photo background. 


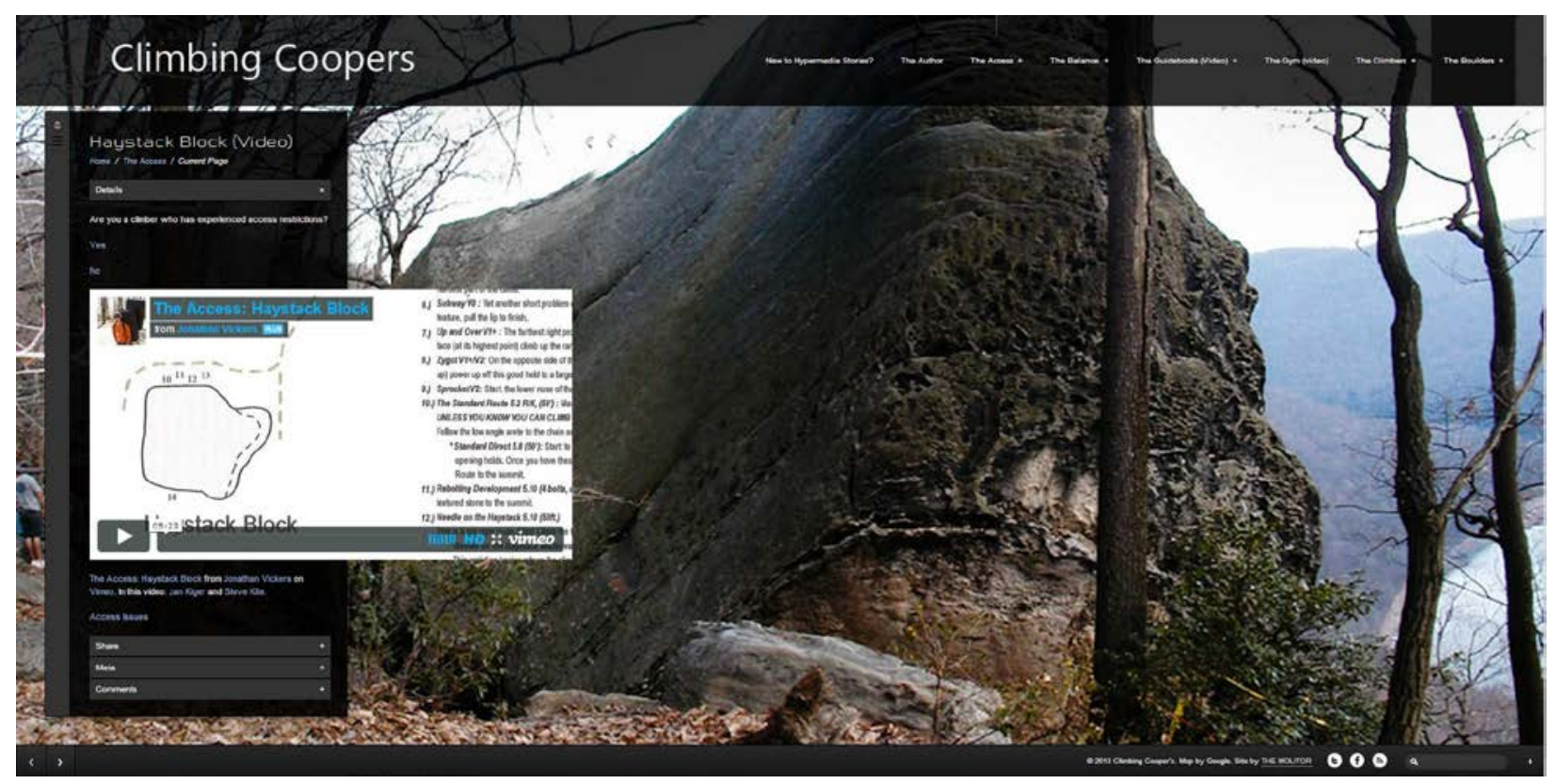

Figure 9. This is an example of a page containing a video component. The background is a still image of the Haystack Boulder and the video tells the story about the fact that it is shifting and climbing is no longer allowed on it.

Still photographs were used throughout the site in two ways. The first was the use of photographs as backgrounds on the various pages (see figure 9) and the second was in the form of galleries that are included within a drop-down menu found in the text box on the left of a given page (see figure 10). These gallery options were part of the original theme, but I was unable to include captions for the photo in the galleries. I was able to work with coder Alex Yohn to allow for the "title" of those images to be show below the picture being viewed in the gallery. I was able to include any caption information I wanted to display in this "title" field on the back end and it would display below the photo being viewed in the gallery (see figure 10). 


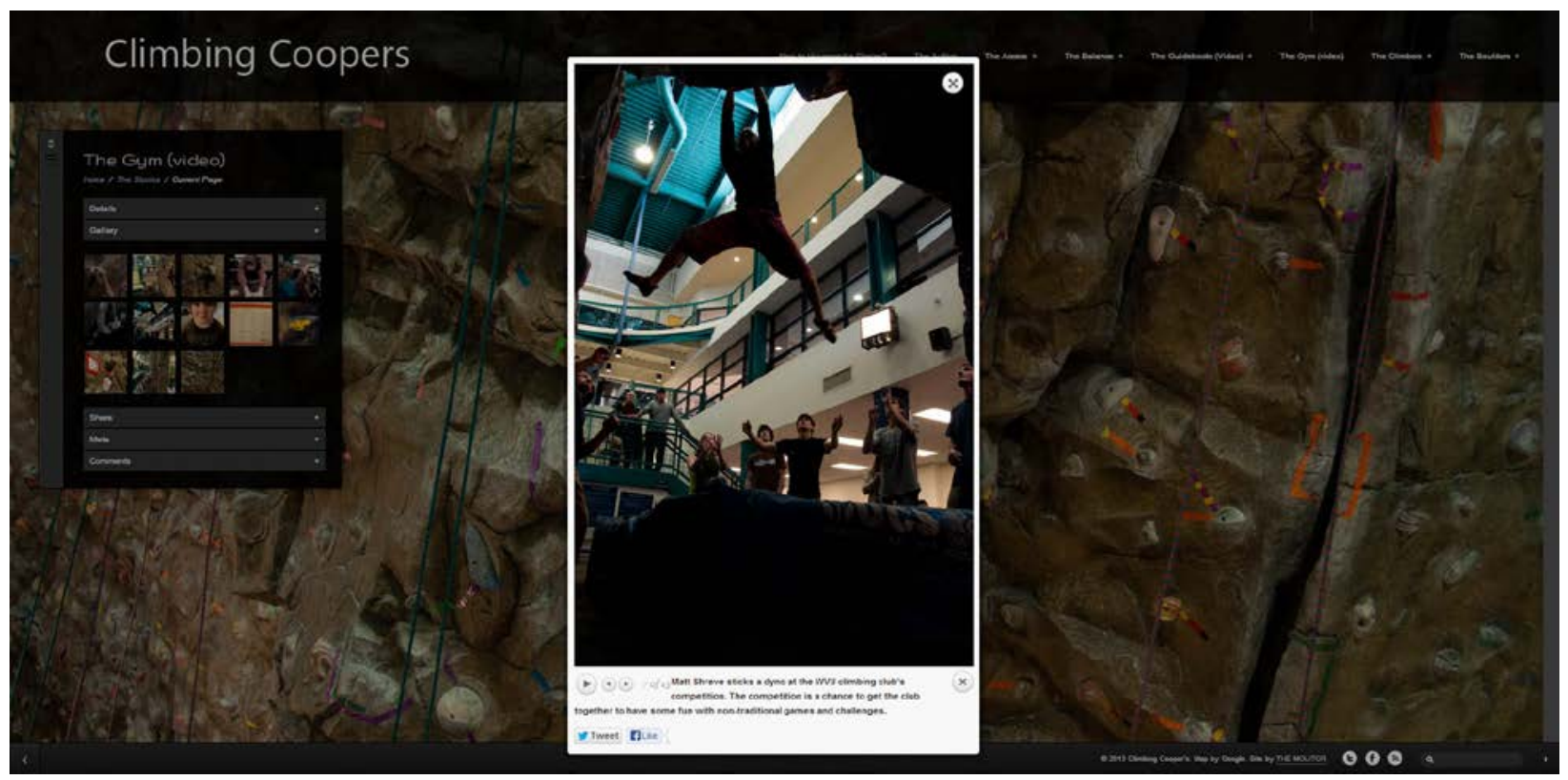

Figure 10. This screen capture of The Gym page shows an example of a photo gallery. To the left is a grid of thumbnails showing the images and chosen images can be viewed larger in the center with caption info found below it.

Climbing Coopers had several sections that are primarily text. This includes many of the bios and area descriptions. Hyperlinks were heavily incorporated to link these texts to other pages and posts throughout the website and even to external content when relevant (see figure 11). Hyperlinks or some way of connecting content fragments is an essential part of hypermedia (Geest, 1994; McEneaney, 2009). A hypertext's links between content fragments or nodes allow for a non-linear experience and vary a reader's navigation options (Geest, 1994; McEneaney, 2009). The use of hyperlinks was important links to internal pages and posts open in the same window assuming a reader's intent was to move on to a new story fragment. External hyperlinks are opened in new windows because many of these links were additions and elaborations to the story fragment being viewed. This allows a reader to easily return to their place on the Climbing Coopers website after viewing the additional information from the external site. 


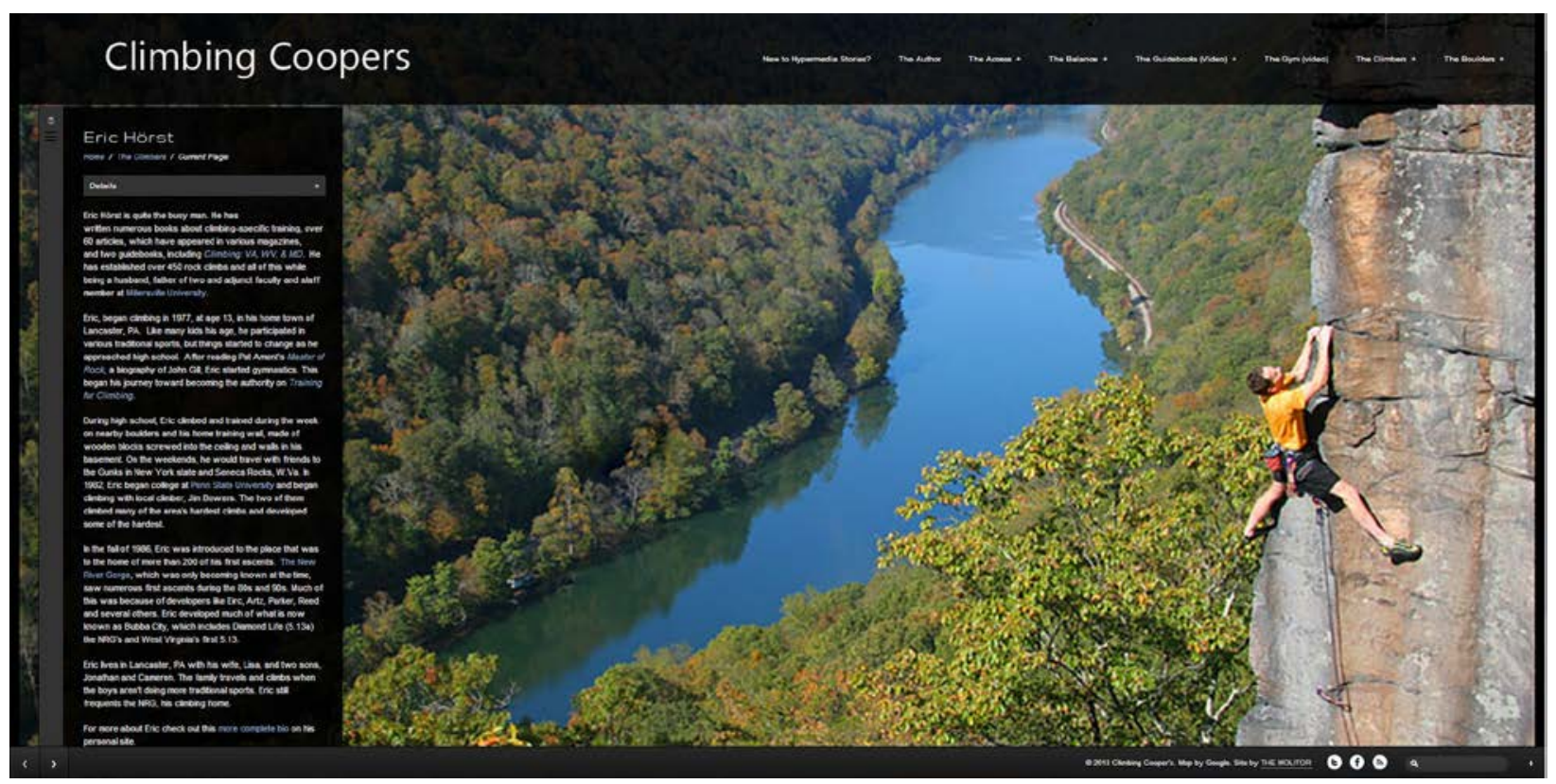

Figure 11. This is the bio page for Eric Horst, a known climbing route developer and author of numerous books about training for climbing. It shows a background image of Eric climbing one of his rock climbs found in the New River Gorge, WV. The text at the left describes his position in the climbing community and how he relates to Coopers Rock. Hyperlinks link to various other pages on the site.

Another hurdle relating to the Wordpress theme was the built-in gallery options. The Navigator theme did not display individual captions for the photos within that gallery (see figure 12). I believe this was the greatest weakness of the theme and I couldn't understand why any gallery would not include an option for captions. After some forum discussions with the theme developer known as the Molitor and Alex Yohn, it was possible to do some minor adjustments to the HTML through the back end allowing the information in the title field of each image to show up as a caption in the galleries. I then went back and moved each caption to the "title” field. 


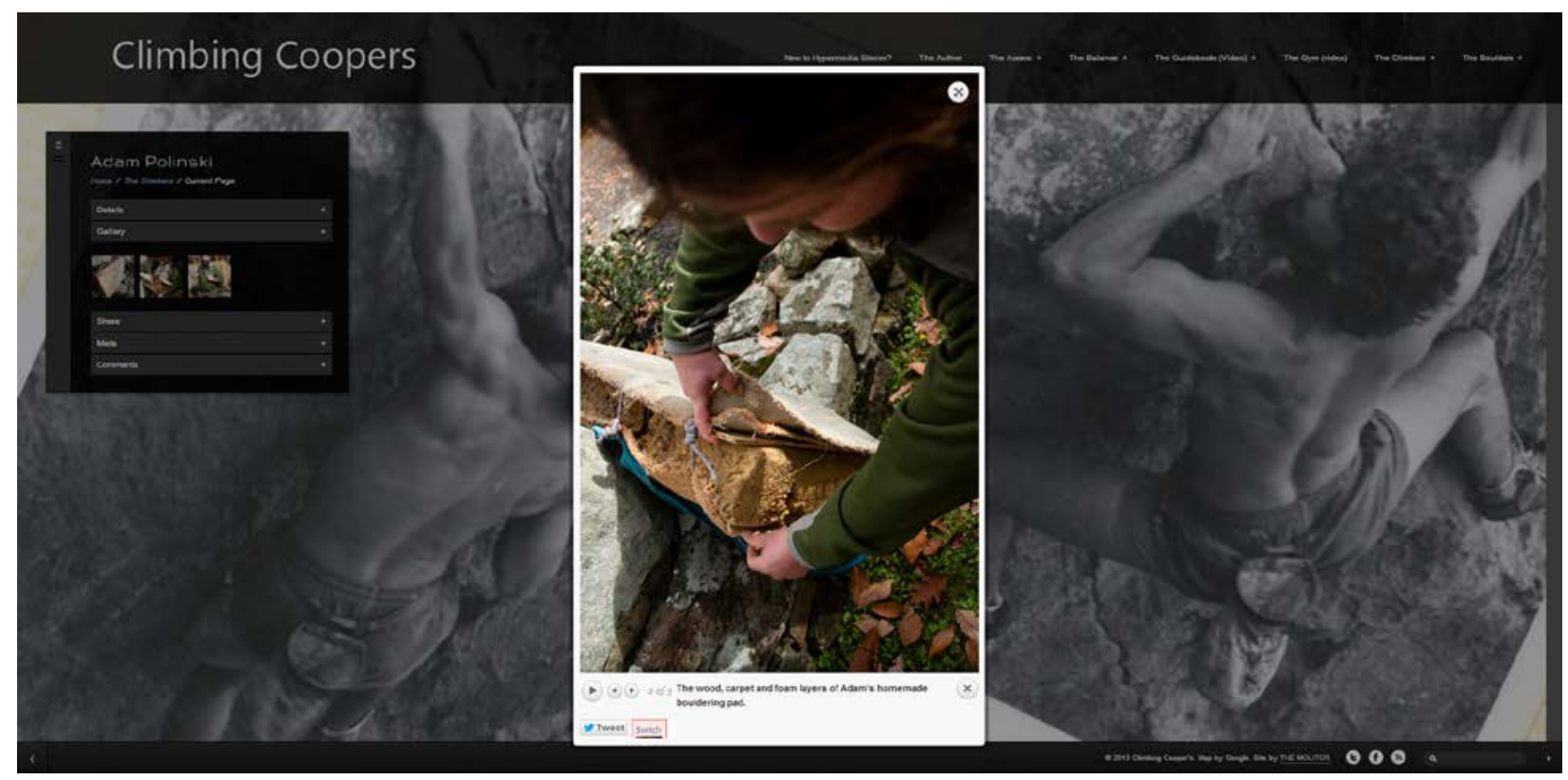

Figure 12. This is a photo in the gallery on Adam Polinski's bio page. A caption about the homemade bouldering pad is visible below the photo thanks to some small edits in the Navigator Themes code.

This project used several common software products and free or inexpensive online tools for this project. This was because of limited access to financial and coding resources. The hope was to demonstrate the power of the tools available to most journalists, with or without any background in coding. Microsoft Word as used for word processing and nearly all of the editing was done using common Adobe products such as Photoshop, Lightroom and Premiere. I purchased the entire Adobe Suite through Adobe’s cloud subscription. I hosted my site through Bluehost, a web hosting company, and purchased the Navigator theme from Theme Forest.

\section{Prompting}

Since the subject matter of climbing is often jargon-heavy and could be hard to conceptualize by non-climbers, some propose the benefits of including prompts to help those with less subject knowledge (Lawless, 2007; McEneaney, 2009). These prompts are intended to fill in any missing information that might be necessary to understanding the content. Those who are already familiar with the subject, in this case the climbers, can skip the additional content or 
receive different content that pertains more to them. The ability for a high-knowledge reader to bypass the additional information is important because it has been proposed that these elements could increase the cohesion and linearity of the story. McNamara (2005) suggests that highknowledge readers can actually benefit from a lack of cohesion making these segments detrimental to their experience.

On the Climbing Coopers website, these prompts are generally found at the beginning of the text box found on the left side (see figure 13). When the reader answers the question the appropriate content for that reader appears in a pop up window in the center. The image below shows a prompt found on the page discussing the publication of the bouldering guide (see figure 13). It is intended to allow climbers to bypass any unnecessary explanation while allow those who may have never used a guidebook to get additional explanation need to understand the story. This example demonstrates the ability to provide both high and low knowledge readers with relevant information.

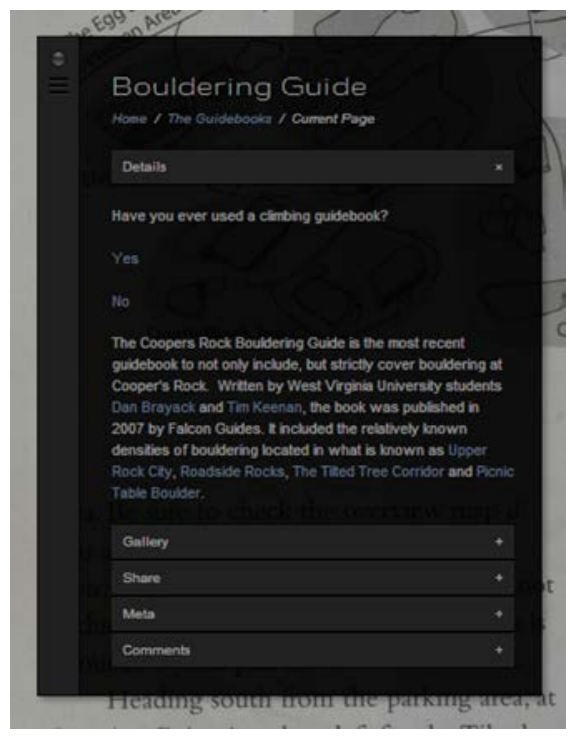

Figure 13. This is a prompt about guidebooks on the Climbing Coopers website. 


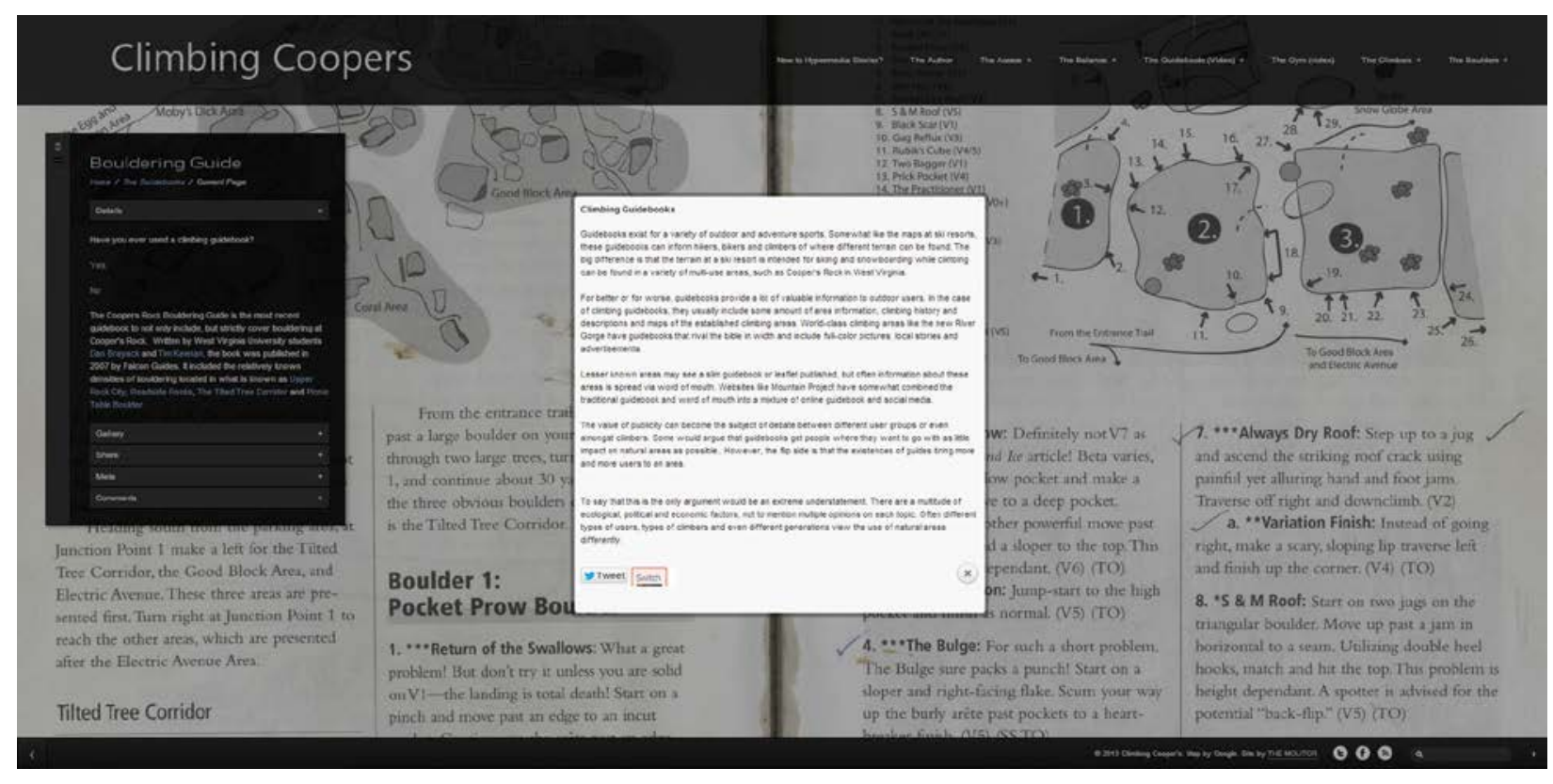

Figure 14. This is the pop up box a reader who picked "No" to the question, "Have you ever used a climbing guidebook?"

In this example if the reader says, "No,” they will receive a pop up box with more information describing the use and need for a guidebook and any details about them that might be pertaining (see figure 14 and 15). If the reader said, "Yes” they were linked to an Amazon page where they could look at and buy the current bouldering guidebook to Coopers Rock (see figure 16). 


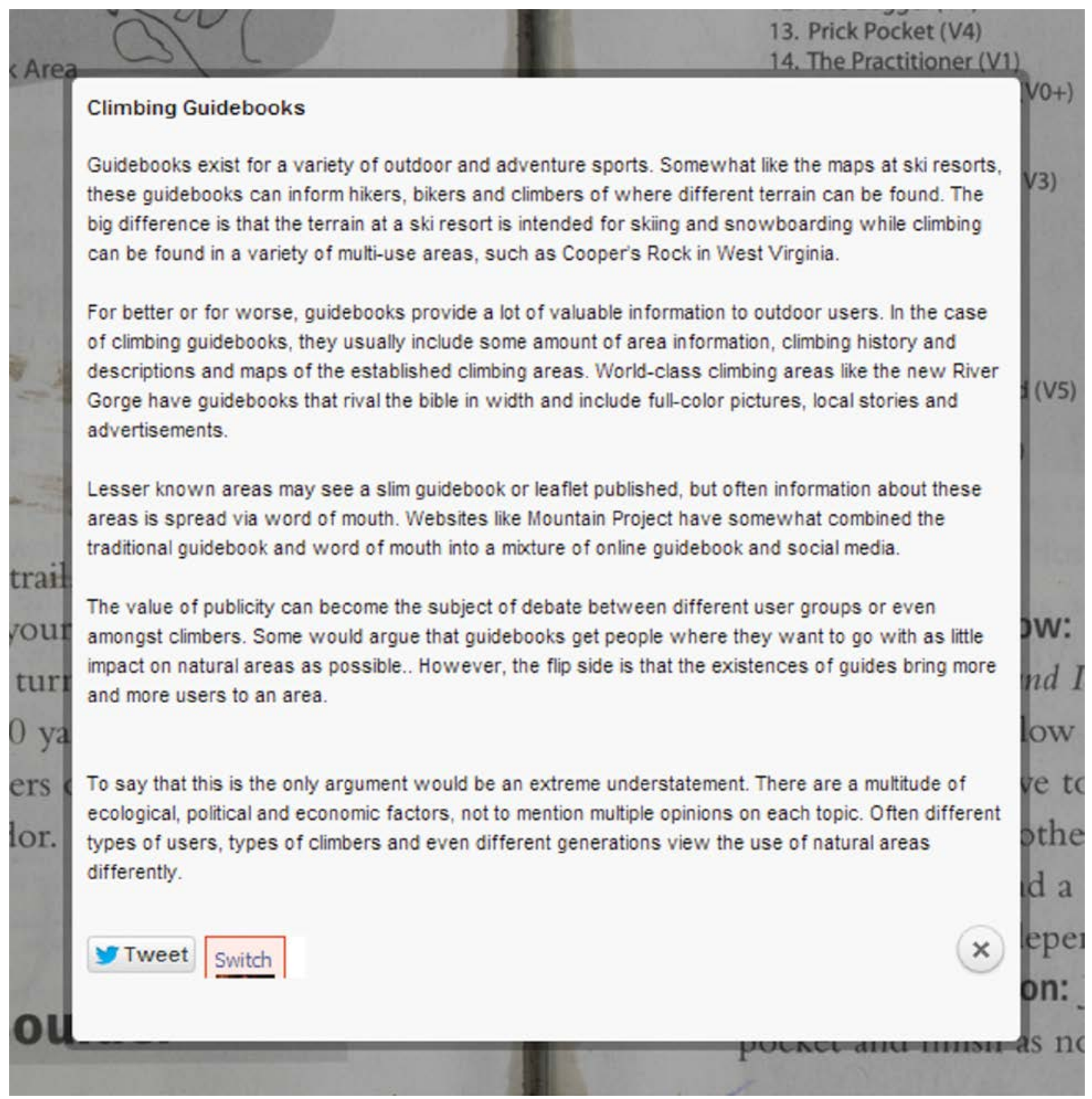

Figure 15. A closer look at the pop up box a reader might get on the guidebooks page. 


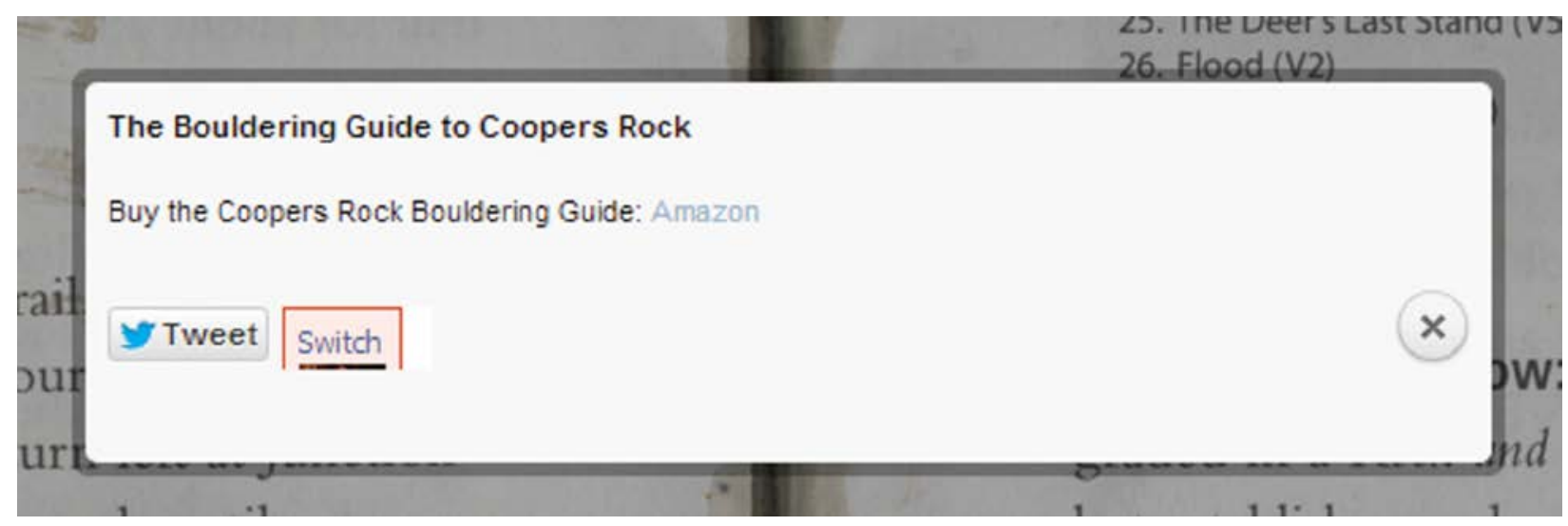

Figure 16. The pop up box a reader would get if they had said "Yes" to the question, "Have you ever used a climbing guidebook?”

\section{Discussion and Conclusion}

The goal of the site is not that users learn anything particular, but that they walk away with an understanding of the dynamic and interrelated nature of the various components making up the climbing community surrounding Coopers Rock State Forest. Not only was this story a good fit for hypermedia, but it would seem that most community stories, given their complex relationships between story elements such as people, places, events and issues, lend themselves to this type of format.

Some studies have suggested that readers of hypermedia stories attain better knowledge transfer of higher order cognitive skills (Mesbah, 2006; Jacobson and Spiro, 1995; Tremayne, 2003). I would argue that understanding complex social issues and dynamic communities requires higher order cognitive skills. It is important to reiterate that this format is not shown to be beneficial for those learning information that is memory-oriented (Tremayne, 2003; Jacobson and Spiro, 1995). When the content stresses factual memorization, a simpler more linear format is better for learning whereas more complex formats may allow for a more complex and flexible understanding of the information. Jacobson and Spiro (1995) showed this with study showing 
that a hypertext format better prepared a group for an essay test where a simpler more linear format prepared another group better for short answer questions.

Other studies have shown reader characteristics to play a role in the success of hypermedia formats; those with more prior knowledge having greater gains from its use (Salmeron, 2006). This important caveat suggests that hypermedia is only incrementally beneficial for those with prior knowledge of the information (Lowrey, 2006). In an effort to gain benefits from the medium while avoiding the pitfall of losing readers unfamiliar with the topic, I tried to control for the prior knowledge discrepancy with prompts.

\section{Audience Considerations and Prompting}

The most innovative aspect of my site from a multimedia perspective is the inclusion of prompts meant to maximize reader experiences within a diverse user group. This group would have a varied understanding of both the hypermedia format and the subject matter. I expected that a variety of audience types and several different user groups will visit my site, including local climbers, non-local climbers and locals with no climbing experience. These and any other additional reader types will have completely different levels of prior knowledge. If used, the prompts should help these different readers fill in any contextual gaps between content chunks.

Given McNamara’s (2001, 2005) research suggesting a relationship between prior knowledge and cohesion, I wanted to make certain that those with prior knowledge were not forced to consume the content in a linear and/or predetermined sequence. I had hopes they could explore the content in any way that they chose based on personal interest, prior knowledge and aesthetics. However, I didn’t want this lack of cohesion to lead to disorientation in my lessknowledgeable readers as predicted by the literature (McDonald \& Stevenson, 1996; Stanton, 2009). McNamara (2001, 2009) has demonstrated that high-knowledge readers benefit from low- 
cohesion texts while low-knowledge readers benefit from highly cohesive texts. This project was an attempt to cater to both types of readers.

My plan was to include optional content or prompts intended on helping increase a nonclimber's subject knowledge while not providing unnecessary content to those with climbing experience. These were meant to act like pre-reading activities which have been shown to increase a reader's prior knowledge and help them with nonlinear engagement (Lawless, 2007). Much of the additional information, which I provided through the use of prompting questions, is meant to increase a reader's knowledge of the subject matter while the prompt found in the navigation bar is meant to increase a reader's familiarity with the format of hypermedia.

Beyond increasing both the format and content knowledge of readers, I also included some additions that might be more associated with adjusting the stance or orientation of a reader as suggested by McEneaney (2009). While a low-knowledge reader would most likely get additional subject knowledge from the prompts found on the Access page and various boulder pages, a high-knowledge reader is presented with information about the activism organization known as Access Fund or a quote from local climber Adam Polinski about how he views the sport of bouldering.

\section{Project Observations and Reflections}

There are several important takeaways from this project, some in regards to prompting or pre-reading while others are simply lessons about hypermedia design and storytelling. In many situations, additional prompts for low-knowledge readers are unnecessary when hyperlinks can be better integrated into the content and more and more readers understand their functionality. However, I do think that more complex topics requiring an understanding of multiple perspectives or concepts merit pre-reading activities for those unfamiliar with the subject matter. 
Preparing a reader for the format being used and frontloading their reading to maximize their experience may be even more important. Those creating hypermedia stories should consider the entire range of prompts, links and other ways of customizing their content for different readers. Tailoring this content can include using prompts or links to increase a reader's subject knowledge, format understanding or adjusting their reading goals and orientation toward the story or topic (Lawless, 2007; McEneaney, 2009).

Deciding how to execute and actually executing the prompting were by far the hardest aspect of my project. As to be expected, the Navigator theme didn’t originally have the ability to add modal dialog boxes (pop-up boxes) and the typical WordPress plug-ins didn’t behave properly with the theme. Fortunately Alex was able to make some simple HTML adjustments that allowed the Pretty Photo plugin to display the dialog boxes. However, we were forced to compromise on where the questions determining reader knowledge level would be asked. While the original plan was to include a separate pop-up box for the questions posed on each page, I preferred that readers have the option to skip certain questions now that we were forced to put them in the actual content box. I think if the questions had been in their own pop-up box it would have been annoying and negatively impacted a reader’s experience.

Beyond the information or intended use of the prompts, a hypermedia author should consider the way these prompts, links etc. are implemented and how they will be perceived by all users. My original idea was for these prompts to be pop-up boxes, but I was forced to incorporate these prompts into the content boxes themselves. In the end, I am happy the prompts are not too obtrusive, particularly since I believe the largest user group will be climbers who do not require the additional information. If I believed that more users might be non-climbers, I might want the prompts to be more obvious in order to make certain people understand the material. In future 
projects I will consider both the user types and the number of each type of user. I believe this approach of layered content for different user groups is useful for the storytelling of many complex or polarizing issues. Having the ability to tailor a story to different levels of expertise such as with science or political stories where a variety of reader perspectives can be accounted for is greatly beneficial to conveying information and expressing these stories.

\section{Notes for Publishers, Editors and Content Creators}

Below is a summation of key points that may be beneficial to publishers, editors and content creators. The first list contains suggestions for possible approaches to hypermedia and the use of prompts. The second is a list of potential ideas and possibilities that could benefit future authors when considering the use of hypermedia and/or prompts.

Hypermedia and Prompting Suggestions

- Collect most of your content before finalizing the structure of your hypermedia piece. The two will inform one another and it is helpful to remain flexible in the way you integrate your content into your interface.

- Know all of your technical capabilities and limitations before designing your site or prompts. While it is possible to learn new skills or hire others to provide certain skills, many content creators do not have that luxury. As free online options improve it will be easier and easier for everyone to create hypermedia stories.

- Think of hyperlinks as prompts and consider all of the benefits of using them to engage your reader. You might provide additional information for less knowledgeable readers, allow more curious or literate readers to dive deeper in a subject or provide other perspectives to richen a reader's understanding of a complex topic.

- Consider frontloading complex topics by providing some level of information that can inform readers or affect their stance about the subject. 
- Decide on how visible or prevalent you want your prompts to be. Going overboard could be detrimental to the flow of the story and negatively impact a readers experience with it.

Future Possibilities and Ideas

- Consider using a hypermedia format for organizing preexisting content or blog material. Combining several elements together or linking blog posts can provide a new level of context to each piece of the story.

- Use prompting to gather reader information. This information can be useful for targeted advisements and to provide several storytelling advantages to a content creator. Prompts could provide not only useful feedback about the success of stories, but also provide opportunities for fact checking, reporting and engaging with your audience.

- Consider using prompts to integrate advertisements. The ads on Pandora are a great example of prompts being used to provide sponsor information. Now there are even sponsor activities that allow a user to interact with a brand's content or provide useful info to that company. It may be possible to use certain designs to limit the negative impact ads have on a reader experience. It can even make it fun or add to the story if done well.

- Don't just consider yourself an author, but also a curator of information and a facilitator of an experience. Storytelling will continue to change as technology advances and content creators should consider study in other areas of expertise such as social media and experiential education.

\section{Future Hypermedia Projects}

This method of storytelling is not being used by a variety of publications and organizations despite the value this type of piece could have for a publication such as Rock and Ice or Climbing. It could just as easily be used as a form of content marketing for an organization such as Access Fund or a company like Five Ten (climbing shoe manufacturer). 
While I had planned the entire story out starting with the story and website structure, I see this type of storytelling as an appropriate way to combine many preexisting stories together in an interactive interface. The hypertext story could be an ongoing mosaic of already completed narratives that could be continually added too. A hypermedia story could act as a framework to present already published material in a way that creates new meaning through the context each of the story fragments gives to one another. Looking at hypermedia and its ability to portray multiple perspectives and angles has great potential for complex storytelling especially in regards to polarizing issues. Journalists should be curious about the new tools and technologies that are rapidly emerging and the format(s) that are developing consequently. I hope to see future research in what these formats can do for not only a variety of readers, but what they mean for journalists and their balanced or objective reporting of stories.

Other research as to how hypermedia and related formats are used by readers is also important. Further research is needed to see how specific audiences, such as distinct communities, can be better engaged through hypermedia's use. As things progress I would expect to see these types of stories compared to actual experiences. Experiential education in particular refers case studies, much like hypermedia, and prepares learners for specific experience with what is called frontloading, which could be compared to prompting.

\section{Conclusion}

At the very least the Coopers Rock Project can be looked at as an example of using prompts to provide information for low-knowledge readers. You can also see examples of providing more appropriate information to high-knowledge readers. As technology improves and more and more people have access to tools, we may see more opportunity to implement stories such as this. The biggest hurdle seems to be the amount of time a story like this takes, but I think it can be economical as a means to organize or facilitate collaborative authoring. I think you will 
see them as a way to organize stand-alone components into a navigable selection or as a way to bring several authors together for a story. No matter what form media takes in the future, I expect it to be more and more tailored to a variety of readers or viewers. 


\section{References}

Ayersman, D. J. (1993). An overview of the research on learning styles and hypermedia environments Retrieved from http://search.ebscohost.com/login.aspx?direct=true\&db=eric\&AN=ED356756\&site=ehost$\underline{\text { live }}$

Borsook, T. K., \& Higginbotham-Wheat, N. (1992). A psychology of hypermedia: A conceptual framework for $R \& D$ Retrieved from http://search.ebscohost.com/login.aspx?direct=true\&db=eric\&AN=ED345697\&site=ehost$\underline{\text { live }}$

Fredin, E. S. (1997). Rethinking the news story for the internet: Hyperstory prototypes and a model of the user. Journalism \& Mass Communication Monographs, (163), 1-47. Retrieved from http://search.ebscohost.com.www.libproxy.wvu.edu/login.aspx?direct=true\&db=a9h\&AN= $\underline{9712194557 \& \text { site }=\text { ehost-live }}$

Geest, T. v. d. (1994). Hypertext: Writing and reading in a non-linear medium. In M. Steehouder, C. Jansen, P. v. d. Poort \& R. Verheijen (Eds.), Quality of technical documentation (pp. 49-66). Amsterdam: Rodopi. Retrieved from http://search.ebscohost.com/login.aspx?direct=true\&db=mzh\&AN=1995010202\&site=ehost $\underline{\text {-live }}$

Jacobson, M. J., \& Spiro, R. J. (1995). Hypertext learning environments, cognitive flexibility, and the transfer of complex knowledge: An empirical investigation. Journal of Educational Computing Research, 12(4), 301-33. Retrieved from 
http://search.ebscohost.com/login.aspx?direct=true\&db=eric\&AN=EJ510379\&site=ehost$\underline{\text { live }}$

Kalyuga, Slava (2007). Expertise Reversal Effect and Its Implications for LearnerTailored Instruction. Educational Psychology Review, 19(4), 509-539. Doi:10.1007/s10648007-9054-3

Lawless, K. A., Schrader, P. G., \& Mayall, H. J. (2007). Acquisition of information online: Knowledge, navigation and learning outcomes. Journal of Literacy Research, 39(3), 289-306. doi:10.1080/10862960701613086

Lowrey, W., \& Choi, J. (2006). The web news story and cognitive flexibility. In X. Li, \& X. Li (Eds.), Internet newspapers: The making of a mainstream medium. (pp. 99-117). Mahwah, NJ US: Lawrence Erlbaum Associates Publishers. Retrieved from http://search.ebscohost.com/login.aspx?direct=true\&db=psyh\&AN=2006-05719$\underline{006 \& \text { site }=\text { ehost-live }}$

Lowrey, W., \& Kim, K. S. (2009). Online news media and advanced learning: A test of cognitive flexibility theory. Journal of Broadcasting \& Electronic Media, 53(4), 547-566. doi:10.1080/08838150903323388

Mancini, C., \& Scott, D. R. (2006). Hyper-document structure: Representing cognitive coherence in non-linear documents. Information Design Journal (IDJ), 14(3), 232-235. Retrieved from http://search.ebscohost.com/login.aspx?direct=true\&db=ufh\&AN=23333280\&site=ehost$\underline{\text { live }}$

Massey, B. L. (2004). Examination of 38 web newspapers shows nonlinear storytelling rare. Newspaper Research Journal, 25(3), 96-102. Retrieved from 
http://search.ebscohost.com/login.aspx?direct=true\&db=ufh\&AN=14598434\&site=ehost-

live

Mesbah, H. M. (2006). The impact of linear versus nonlinear listening to radio news on recall and comprehension. Journal of Radio Studies, 13(2), 187-200.

doi:10.1080/10955040701313222

McDonald, S., \& Stevenson, R. J. (1996). Disorientation in hypertext: The effects of three text structures on navigation performance. Applied Ergonomics, 27(1), 61-68. Retrieved from http://search.ebscohost.com/login.aspx?direct=true\&db=cmedm\&AN=15676313\&site=ehos t-live

McEneaney, J. E., Li, L., Allen, K., \& Guzniczak, L. (2009). Stance, navigation, and reader response in expository hypertext. Journal of Literacy Research, 41(1), 1-45. doi:10.1080/10862960802695081

McNamara, D. S. (2001). Reading both high-coherence and low-coherence texts: Effects of text sequence and prior knowledge. Canadian Journal of Experimental Psychology/Revue Canadienne De Psychologie Expérimentale, 55(1), 51-62. doi:10.1037/h0087352

McNamara, D. S., \& Shapiro, A. M. (2005). Multimedia and hypermedia solutions for promoting metacognitive engagement, coherence, and learning. Journal of Educational Computing Research, 33(1), 1-29. Retrieved from http://search.ebscohost.com/login.aspx?direct=true\&db=eric\&AN=EJ733956\&site=ehostlive; $\underline{\text { http://baywood.metapress.com/link.asp?target=contribution\&id=7N6RPCJLUMHKRYPJ }}$ 
Salmeron, Ladislao, Canas,Jose J., Kintsch, W., \& Fajardo, I. (2005). Reading strategies and hypertext comprehension. Discourse Processes: A Multidisciplinary Journal, 40(3), 171-191. Retrieved from http://search.ebscohost.com/login.aspx?direct=true\&db=eric\&AN=EJ758808\&site=ehostlive; http://dx.doi.org/10.1207/s15326950dp4003_1

Salmerón, L., Kintsch, W., \& Cañas, J. (2006). Coherence or interest as basis for improving hypertext comprehension. Information Design Journal (IDJ), 14(1), 45-55. Retrieved from http://search.ebscohost.com.www.libproxy.wvu.edu/login.aspx?direct=true\&db=ufh\& $\underline{\text { AN }=21327151 \& \text { site }=\text { ehost-live }}$

Stanton, D. (2009). EyeTracking the news: The report of EyeTrack 07A study of print and online reading. Conference Papers -- International Communication Association, , 1. Retrieved from http://search.ebscohost.com/login.aspx?direct=true \&db=ufh\&AN=45287068\&site=ehost$\underline{\text { live }}$

Strobel, J., Jonassen, D. H., \& Ionas, I. G. (2008). The evolution of a collaborative authoring system for non-linear hypertext: A design-based research study. Computers \& Education, 51(1), 67-85. Retrieved from http://search.ebscohost.com/login.aspx?direct=true\&db=eric\&AN=EJ794659\&site=ehostlive; http://dx.doi.org/10.1016/j.compedu.2007.04.008

Tremayne, M. (2003). Learning on the web: The importance of interactivity and motivation International Communication Association. doi:ica_proceeding_12071.PDF 